OPEN ACCESS

Edited by:

Thomas Fath,

Macquarie University, Australia

Reviewed by:

Simone Di Giovanni, Imperial College London,

United Kingdom

Stefania Raimondo,

University of Turin, Italy

${ }^{*}$ Correspondence:

Petr Dubový

pdubovy@med.muni.cz

Received: 08 October 2018 Accepted: 14 January 2019 Published: 04 February 2019

Citation:

Dubový P, Klusáková I, Hradilová-Svĩzenská I, Brázda V,

Kohoutková M and Joukal M (2019) A

Conditioning Sciatic Nerve Lesion

Triggers a Pro-regenerative State in

Primary Sensory Neurons Also of Dorsal Root Ganglia Non-associated

With the Damaged Nerve.

Front. Cell. Neurosci. 13:11. doi: 10.3389/fncel.2019.00011

\section{A Conditioning Sciatic Nerve Lesion Triggers a Pro-regenerative State in Primary Sensory Neurons Also of Dorsal Root Ganglia Non-associated With the Damaged Nerve}

\author{
Petr Dubový*, Ilona Klusáková, Ivana Hradilová-Svíženská, Václav Brázda, \\ Marcela Kohoutková and Marek Joukal \\ Department of Anatomy, Laboratory of Cellular and Molecular Neurobiology, Faculty of Medicine, Masaryk University, \\ Brno, Czechia
}

The primary sensory neurons of dorsal root ganglia (DRG) are a very useful model to study the neuronal regenerative program that is a prerequisite for successful axon regeneration after peripheral nerve injury. Seven days after a unilateral sciatic nerve injury by compression or transection, we detected a bilateral increase in growth-associated protein-43 (GAP-43) and superior cervical ganglion-10 (SCG-10) mRNA and protein levels not only in DRG neurons of lumbar spinal cord segments (L4-L5) associated with injured nerve, but also in remote cervical segments (C6-C8). The increase in regeneration-associated proteins in the cervical DRG neurons was associated with the greater length of regenerated axons 1 day after ulnar nerve crush following prior sciatic nerve injury as compared to controls with only ulnar nerve crush. The increased axonal regeneration capacity of cervical DRG neurons after a prior conditioning sciatic nerve lesion was confirmed by neurite outgrowth assay of in vitro cultivated DRG neurons. Intrathecal injection of IL-6 or a JAK2 inhibitor (AG490) revealed a role for the IL-6 signaling pathway in activating the pro-regenerative state in remote DRG neurons. Our results suggest that the pro-regenerative state induced in the DRG neurons non-associated with the injured nerve reflects a systemic reaction of these neurons to unilateral sciatic nerve injury.

Keywords: unilateral nerve injury, primary sensory neurons, pro-regenerative state, GAP-43, SCG-10, IL-6, ulnar nerve crush, neurite outgrowth assay

\section{INTRODUCTION}

It is well-known that besides extrinsic factors, activation of a neuronal regenerative program is necessary for successful axon regeneration after peripheral nerve injury. The primary sensory neurons, whose bodies are in the dorsal root ganglia (DRG), are a useful model to study the mechanisms regulating the neuronal regeneration program after axotomy. The DRG neurons have peripheral and central branches of afferent axons with different responses to injury. Injury to peripheral axonal branches induces transcription-dependent changes of regeneration-associated 
genes and proteins that promote axon regeneration by enhancing the regeneration potential of DRG neurons (Liu et al., 2011). In contrast, central axonal branches extending into the dorsal columns of the spinal cord fail to regenerate when injured because insufficient activation of the neuronal regeneration program (Schwaiger et al., 2000; Qiu et al., 2005). However, a conditioning lesion of the peripheral nerve, where peripheral axonal branches are injured beforehand, triggers a regenerative program in DRG neurons that is sufficient to allow regeneration also of central axonal branches (Neumann and Woolf, 1999). This phenomenon of a conditioning peripheral nerve lesion with the activation of the pro-regenerative state in DRG neurons is at least partly associated with upregulation of some neuropoietic cytokines including IL-6 (Cafferty et al., 2001; Zigmond, 2011, 2012). It was also shown that increased axon regeneration was conditioned in the homologous nerve contralateral to the injured nerve (Yamaguchi et al., 1999; Ryoke et al., 2000).

In our previous experiments we have found bilateral increased levels of IL6, as well as its receptor mRNA and protein, not only in DRG associated with the injured sciatic nerve, but also in remote cervical DRG (Brázda et al., 2013; Dubový et al., 2013). Moreover, unilateral sciatic nerve injury induced bilateral activation of STAT3 by phosphorylation at the tyrosine-705 (Y705) position in DRG neurons of both lumbar and cervical segments (Dubový et al., 2018a) that is a critical transforming factor of the neuronal pro-regenerative state (Bareyre et al., 2011; Zigmond, 2011). Based on our own and other previously published results, we hypothesize that nerve injury may stimulate the pro-regenerative state not only in the DRG neurons associated with damaged axons, but also in DRG neurons not directly associated with the injured nerve.

Activation of the neuronal pro-regenerative state is characterized by upregulation of various transforming factors, regeneration-associated genes and proteins which are important intrinsic determinants of neuronal regeneration capacity (Mar et al., 2014; Rishal and Fainzilber, 2014; Chandran et al., 2016). For example, phosphorylation and activation of the transcription factor cJun (Itoh et al., 2009; Frey et al., 2015) and the mitogenactivated protein kinase p38 (p38 MAPK; Verma et al., 2005; Nix et al., 2011) is associated with induction of the pro-regenerative state in DRG neurons following nerve injury.

Regeneration-associated proteins like growth-associated protein-43 (GAP-43) or superior cervical ganglion-10 (SCG-10) are generally used as markers for detecting the neuronal pro-regenerative state (Bonilla et al., 2002; Mason et al., 2002). GAP-43 is the prototypical GAP, is expressed at high levels in neurons during development and concentrated in the axonal growth cone (Skene and Willard, 1981). DRG neurons of naive rats display a low basal level of GAP-43 that is significantly increased after nerve injury (Stewart et al., 1992; Schreyer and Skene, 1993; Liabotis and Schreyer, 1995). The SCG-10 protein, also known as stathmin 2, is a neuron-specific member of the stathmin family (Sugiura and Mori, 1995) that is upregulated specifically in primary sensory neurons regenerating their axons (Shin et al., 2014).
The goal of the present study was to investigate whether a sciatic nerve injury can activate the pro-regenerative state of DRG neurons not only in the corresponding lumbar but also in remote cervical spinal cord segments. The pro-regenerative state of DRG neurons was shown by the upregulation of GAP-43 and SCG-10 mRNAs and proteins as well as activation of cJun and p38 in correlation with the extent of regenerated axons after ulnar nerve crush following prior sciatic nerve injury. Increased axon regeneration capacity in cervical DRG neurons initiated by the conditioning sciatic nerve lesion was also confirmed by neurite outgrowth assay of in vitro cultivated DRG neurons. Moreover, intrathecal injection of IL-6 revealed that this cytokine can mediate this systemic reaction of DRG along the neuroaxis after unilateral sciatic nerve lesion.

\section{MATERIALS AND METHODS}

\section{Animals and Surgical Treatment}

The experiments were performed in 194 adult male rats (Wistar, 250-280 g, Anlab, Brno, Czechia) housed in $12 \mathrm{~h}$ light/dark cycles at a temperature of $22-24^{\circ} \mathrm{C}$ under specific pathogen-free conditions in the animal housing facility of Masaryk University. Sterilized standard rodent food and water were available ad libitum. Animals for surgical treatments were anesthetized using a mixture of ketamine $(40 \mathrm{mg} / \mathrm{ml})$ and xylazine $(4 \mathrm{mg} / \mathrm{ml})$ administered intraperitoneally $(0.2 \mathrm{ml} / 100 \mathrm{~g}$ body weight $)$. All surgical procedures were carried out under sterile conditions by the same person according to protocols approved by the Animal Investigation Committee of the Faculty of Medicine, Brno, Czechia.

The right sciatic nerve of rats was exposed in mid-thigh, ligated with two ligatures and cut (complete sciatic nerve transection, CSNT). The proximal nerve stump was fixed in muscles to protect the distal stump from reinnervation. A longitudinally slit silicone tube of $2-\mathrm{mm}$ length and $1 \mathrm{~mm}$ internal diameter was placed around the right sciatic nerve of rats to reduce the nerve diameter (sciatic nerve compression, SNC; Schmid et al., 2013). The tube was tied in place with a sterile thread to close and prevent tube from opening. The muscles and skin were closed with 5/0 sutures. The right sciatic nerve of sham-operated rats was carefully exposed without any lesion.

To determine the progression in time of the pro-regenerative state in cervical DRG after sciatic nerve lesion, a set of SNC-operated rats from the pilot study was left to survive for 1 , 3,7 , and 14 days and compared with naïve rats or sham-operated rats surviving for 1 and 3 days ( $n=3$ for each group). Based on the pilot study in which GAP-43 peaked at 7 days after unilateral sciatic nerve lesion (Figure 1), further groups of operated and sham-operated rats were left to survive for 7 days after surgical treatment and used for bilateral analysis of the neuronal regeneration program in both lumbar and cervical DRG.

\section{Quantitative Immunohistochemical Analysis}

Naïve rats and sham-, SNC- and CSNT-operated rats $(n=6$ for each group) were deeply anesthetized with a lethal dose of 

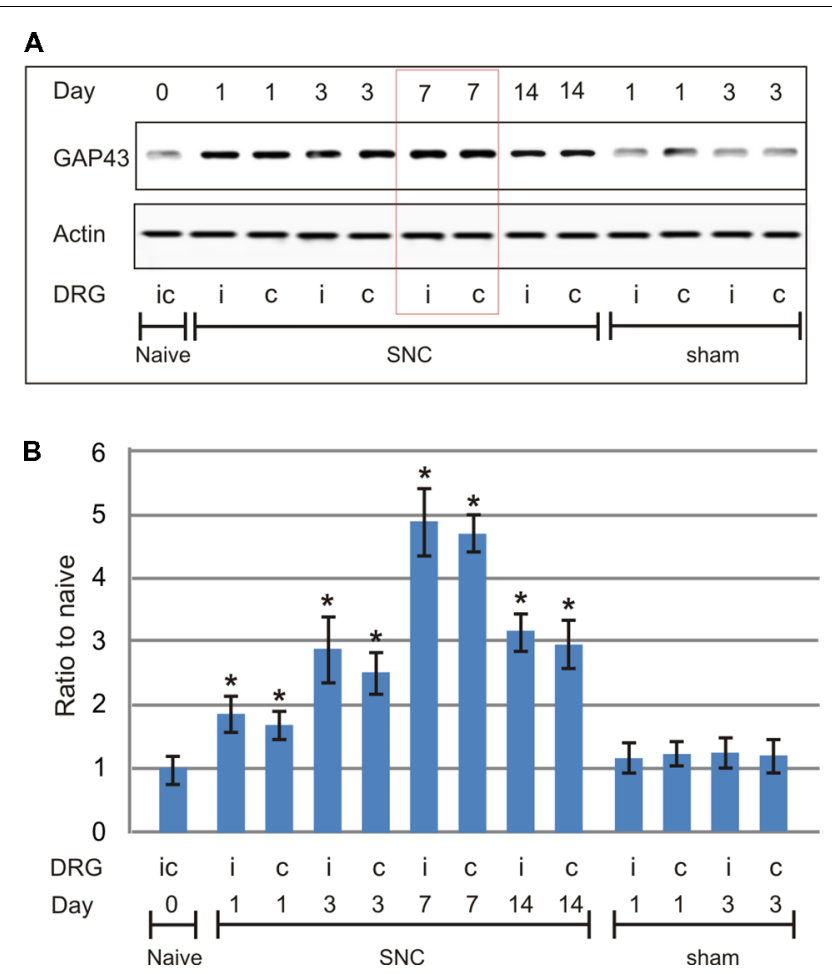

FIGURE 1 | Results of western blot analysis of growth-associated protein-43 (GAP-43) protein levels in cervical dorsal root ganglia (DRG; C6-C8) removed from naïve rats as well as sham- and sciatic nerve compression (SNC)-operated rats. Sham-operated rats were left to survive for 1 and 3 days, SNC-operated rats for $1,3,7$ and 14 days $(n=3$ for each group). Upper panel (A) shows a representative western blot with GAP-43 protein in cervical DRG ipsilateral (i) and contralateral (c) to unilateral SNC. Equal loading of proteins was confirmed by actin levels (Actin). Lower panel (B) shows densitometry of the individual protein bands after normalization to actin; the intensities of the bands from naïve cervical DRG were as arbitrarily set to $1 . *$ Significant difference $(p<0.05)$ compared to naïve or sham-operated rats in a Mann-Whitney U-test.

sodium pentobarbital (70 mg/kg body weight, i.p.) and perfused transcardially with $500 \mathrm{ml}$ phosphate-buffered saline (PBS, $\mathrm{pH}$ 7.4) followed by $500 \mathrm{ml}$ of Zamboni's fixative (Zamboni and Demartin, 1967). The L4-L5 and C6-C8 DRG from both sides were detected in their intervertebral foramina following total laminectomy and foraminotomy. The DRG were removed, immersed separately in Zamboni's fixative at $4^{\circ} \mathrm{C}$ overnight, and then collected separately into samples of ipsilateral (L-DRGi) and contralateral (L-DRGc) lumbar as well as ipsilateral (C-DRGi) and contralateral (C-DRGc) cervical DRG for each group of rats (naïve, sham-, SNC-, and CSNT-operated).

The DRG samples were washed in $20 \%$ phosphate-buffered sucrose for $12 \mathrm{~h}$, blocked in Tissue-Tek ${ }^{\circledR}$ OCT compound (Miles, Elkhart, IN, USA) and cut to prepare serial longitudinal cryostat sections $(12 \mu \mathrm{m})$. The DRG sections were mounted on chrome-alum covered slides and processed for indirect immunohistochemical staining for GAP-43 and SCG-10. Briefly, DRG sections of lumbar and cervical segments of naïve, sham-, SNC- and CSNT-operated rats were immunostained simultaneously under the same conditions. Sections were washed with PBS containing 0.05\% Tween 20 (PBS-T) and $1 \%$ bovine serum albumin (BSA) for $10 \mathrm{~min}$, treated with $5 \%$ normal donkey serum in PBS-T for $30 \mathrm{~min}$, then incubated with $25 \mu \mathrm{l}$ of mouse monoclonal antibody against GAP-43 (1:500; Sigma, Ronkonkoma, NY, USA) and rabbit polyclonal antibody against SCG-10 (1:1,000; LSBio, Seattle, WA, USA), phospho-cJun (1:100; Cell Signaling, New York, NY, USA) or phospho-p38 (1:200; Chemicon Int., Temecula, CA, USA) in a humid chamber at room temperature $\left(21-23^{\circ} \mathrm{C}\right)$ for $12 \mathrm{~h}$. The immunohistochemical reaction was visualized by treatment with FITC-conjugated and affinity-purified donkey anti-mouse or anti-rabbit secondary antibody (1:100; Millipore, USA) for $90 \mathrm{~min}$ at room temperature. The control sections were incubated without the primary antibody. Sections were stained with Hoechst 33342 to detect cell nuclei, mounted in aqueous mounting medium (Vectashield; Vector Laboratories, Burlingame, CA, USA) and analyzed using an epifluorescence microscope (Nikon Eclipse, Nikon, Czechia) equipped with a camera (DFC-480; Leica Microsystems) and a stabilized power supply for the lamp housing.

The neuronal diameter, GAP-43 and SCG-10 immunofluorescence intensities were measured using a NIS-Elements image analysis system (Nikon, Czechia) as previously (Dubový et al., 2002, 2013). At least 100 neuronal profiles containing nuclei were measured for each animal group. The sizes of DRG neurons in sections for immunofluorescence were categorized as small $(<25 \mu \mathrm{m})$, medium $(25-40 \mu \mathrm{m})$, and large $(>40 \mu \mathrm{m})$ according to their diameters calculated from the areas of neuronal profiles. The immunofluorescence intensities were expressed as mean intensity $\pm \mathrm{SD}$.

\section{Western Blot Analysis}

In the pilot study, the cervical DRG (C6-C8) of naïve rats and rats surviving 1 and 3 days after sham-operation and 1, 3, 7 and 14 days after SNC-operation ( $n=3$ for each group) were removed bilaterally, flash-frozen in liquid nitrogen and stored at $-80^{\circ} \mathrm{C}$ until western blot analysis of GAP-43.

The results of GAP-43 and SCG-10 levels obtained by quantitative immunohistochemistry in DRG neurons 7 days after sciatic nerve lesions were verified by western blot analysis. The DRG from lumbar (L4-L5) and cervical (C6-C8) levels ( $n=3$ for each group in three independent experiments) were collected as described under aseptic conditions. Samples of ipsilateral (L-DRGi) and contralateral (L-DRGc) lumbar DRG as well as ipsilateral (C-DRGi) and contralateral (C-DRGc) cervical DRG from each group of rats were flash-frozen in liquid nitrogen and stored at $-80^{\circ} \mathrm{C}$ until analysis.

The DRG samples were homogenized in PBS containing protease inhibitors (LaRoche, Switzerland) with $0.1 \%$ Triton X100 , and centrifuged at $10,000 \mathrm{~g}$ for $5 \mathrm{~min}$ at $4^{\circ} \mathrm{C}$. Proteins were separated by SDS-polyacrylamide gel electrophoresis (Brazda et al., 2006) and transferred to nitrocellulose membranes by electroblotting (BioRad). Blots were blocked using 1\% BSA in PBST (3.2 mM Na $\mathrm{HPO}_{4}, 0.5 \mathrm{mM} \mathrm{KH}_{2} \mathrm{PO}_{4}, 1.3 \mathrm{mM}$ $\mathrm{KCl}, 135 \mathrm{mM} \mathrm{NaCl}, 0.05 \%$ Tween 20, $\mathrm{pH}$ 7.4) for $1 \mathrm{~h}$ and incubated with anti-GAP-43 mouse monoclonal (1:1,000; Sigma, Ronkonkoma, NY, USA), rabbit polyclonal anti-phosphorylated 
S41-GAP43 (1:500; Thermo Fisher Scientific, Waltham, MA, USA) or anti-SCG-10 (1:500; LSBio, Seattle, WA, USA) antibodies overnight. Blots were washed in PBST and incubated with peroxidase-conjugated anti-mouse or anti-rabbit IgG (1:1,000; Sigma, Seattle, WA, USA) at room temperature for $1 \mathrm{~h}$. Protein bands were visualized by the ECL detection kit (Amersham, USA) on the chemiluminometer reader LAS-3000 (Fuji, Japan) and analyzed using densitometry image software. The levels of proteins were normalized to the value of naive DRG, which was arbitrarily set as one.

\section{Real Time RT-PCR}

The expression of GAP-43 and SCG-10 mRNAs in DRG was analyzed by real-time PCR (RT-PCR). Whole DRG from each group of rats $(n=3$ for each group in three independent experiments) were removed under aseptic conditions from lumbar (L4-L5) and cervical (C6-C8) segments of both sides, collected as ipsilateral and contralateral samples and stored in RNA later (Thermo Fisher Scientific, Inc., Waltham, MA, USA) at $4^{\circ} \mathrm{C}$. First-strand synthesis was performed using TaqMan ${ }^{\circledR}$ High Capacity RNA-to-cDNA Kit and the quality and concentration evaluated by optical density using NanoDrop. PCR amplification, in triplicate for each sample, was performed using ABI Prism 7300, TaqMan ${ }^{\circledR}$ Gene Expression Master Mix, and TaqMan ${ }^{\circledR}$ Gene Expression Assay Probes FAM ${ }^{\mathrm{TM}}$ (Thermo Fisher Scientific, Inc., Waltham, MA, USA) for the target genes GAP-43 (assay ID-Rn01474579_m1) and SCG-10 (assay ID_Rn00584886_m1). Determinations were made with reference to the reporter gene encoding rat actin (actin, beta-Rn00667869_m1) Endogenous Control (VIC $\left.{ }^{\circledR}\right)$. The polymerase activation step at $95^{\circ} \mathrm{C}$ for $15 \mathrm{~min}$ was followed by 40 cycles of $15 \mathrm{~s}$ at $95^{\circ} \mathrm{C}$ and $60 \mathrm{~s}$ at $60^{\circ} \mathrm{C}$. The validity of the results was checked by running appropriate negative controls (water instead of cDNA by for PCR amplification; omitting reverse transcriptase for cDNA synthesis). Specific mRNA levels were calculated after normalizing to actin mRNA in each sample. Relative expression was determined using the Comparative $\mathrm{Ct}$ Model $(\Delta \Delta \mathrm{Ct})$ with actin as the housekeeping gene. Data were presented as relative mRNA units compared with control values (expressed as fold over naive value).

\section{In vivo Assay of Axon Regeneration in Crushed Ulnar Nerve}

Rats with prior operation on SNC $(n=6)$ or CSNT $(n=6)$ for 7 days were re-operated to expose and mobilize a short segment of the right ulnar nerve. The right ulnar nerve was also mobilized in a control group without any previous sciatic nerve injury $(n=6)$. The ulnar nerve was then crushed using a clamp of a defined force of $1.9 \mathrm{~N}$ for $2 \times 1$ min (Ronchi et al., 2009) under a stereoscopic microscope. The distal margin of the crush injury was indicated with a 10-0 epineurial suture and the skin wound was closed with $5 / 0$ sutures.

To investigate peripheral axon regeneration, the ulnar nerves were removed 1 day after the crush injury following pericardial infusion with Zamboni fixative solution and ulnar nerve samples were fixed by immersion in Zamboni fixative solution overnight. After washing with $10 \%$ sucrose in PBS, longitudinal cryostat sections of $10 \mu \mathrm{m}$ thickness were cut and immunostained for SCG-10, which is a more selective marker of regenerating sensory axons than GAP-43 (Shin et al., 2014). SCG-10 fluorescence intensity was analyzed along the length of the nerve distal to the crush; the regeneration index was determined by measuring the length of the longest SCG-10 decorated axons from the crush site (Abe et al., 2010). The length of SCG-10+ axons was measured by a person blind to the experimental conditions in every third section using a NIS-Elements image analysis system (Nikon, Czechia).

\section{In vitro Assay of Increased Axonal Outgrowth of Cervical DRG Neurons Induced by Sciatic Nerve Lesion}

An in vitro culture of dissociated DRG neurons was prepared according to a modified protocol (Christie et al., 2010). Sham-operated rats $(n=4)$ and rats operated by SNC $(n=4)$ or CSNT $(n=4)$ for 7 days were deeply anesthetized by an intraperitoneal application of sodium pentobarbital $(70 \mathrm{mg} / \mathrm{kg})$ and killed by decapitation. The DRG of lumbar (L4-L5) and cervical (C6-C8) segments were removed bilaterally under aseptic conditions following laminectomy. The samples were collected in ice-cold $\mathrm{Ca}^{2+} / \mathrm{Mg}^{2+}$-free Hank's buffered salt solution (CMF-HBSS, Sigma Aldrich), and the spinal roots and connective tissues were removed.

DRG were then dissociated by incubation in medium containing $0.1 \%$ collagenase type $\mathrm{I}(5,000 \mathrm{U} / \mathrm{ml})$ for $90 \mathrm{~min}$ followed by $0.25 \%$ trypsin/EDTA at $37^{\circ} \mathrm{C}$ for $25 \mathrm{~min}$. The DRG suspension was prepared by triturating through glass pipette tips, washed twice with Dulbecco's Modified Eagle's Medium/Nutrient F-12 Ham (DMEM/F12) supplemented with $10 \%$ fetal bovine serum (FBS; all from Sigma Aldrich) and the suspension was spun for $5 \mathrm{~min}$ at $1,500 \mathrm{rpm}$ at $4^{\circ} \mathrm{C}$. The cells were resuspended and placed into a culture medium of DMEM/F12 supplemented with $2 \mathrm{mM}$ glutamine, $100 \mathrm{U} / \mathrm{ml}$ Penicillin and $100 \mu \mathrm{g} / \mathrm{ml}$ Streptomycin (all from Sigma Aldrich), N2 and B27 (ThermoFisher Sci., diluted according to the manufacturer's instructions). The cells were re seeded at a density of 200 cells on glass cover slips previously coated with Geltrex ${ }^{\circledR}$ (ThermoFisher Sci.).

Cultures were incubated at $37^{\circ} \mathrm{C}$ in a humidified atmosphere containing $5 \% \mathrm{CO}_{2}$ for 2 days, washed in PBS, fixed by Zamboni solution for $20 \mathrm{~min}$ and immunostained with mouse anti- $\beta$ tubulin III primary antibody (Sigma, 1:500) overnight at $4^{\circ} \mathrm{C}$. The cells were then washed in PBS followed by incubation with goat anti-mouse TRITC-conjugated secondary antibody (Life Technologies, 1:1,000) for 90 min. Finally, cells were washed in PBS, stained with Hoechst 33342 to detect cell nuclei and mounted in a Vectashield aqueous mounting medium (Vector Laboratories, Burlingame, CA, USA). Three slides for each experimental group were analyzed under a Nikon Eclipse NI-E epifluorescence microscope equipped with a Nikon DS-Ri1 camera (Nikon, Prague, Czechia) using a $10 \times$ objective by a person blind to the experimental conditions.

To analyze neurite outgrowth and length, digital images of at least 100 randomly selected nucleate neurons per cover slip 
were acquired. Neurites longer than the diameter of the neuronal bodies were analyzed in digital pictures converted to grayscale for better visualization. Neurite outgrowth initiation was quantified as the number of neurites per neuron counted using the Count and Taxonomy module of NIS- Elements software (Nikon, Prague, Czechia). Axonal elongation was analyzed by taking the total length of neurites per neuron when axons of individual neurons were traced and measured using the Neurite Tracer Plugin for Image (Pool et al., 2008). The mean number of neurites per neuron and total neurite length per neuron were calculated from triplicate experiments and data were present as mean \pm SD

\section{In vivo Assay of Axon Regeneration in the Crushed Ulnar Nerve and Changes in the Pro-regenerative State of Cervical DRG Neurons After Intrathecal Injection of IL-6 or JAK2 Inhibitor AG490}

Recombinant rat IL-6 protein (R\&D Systems) was dissolved in artificial cerebrospinal fluid (ACSF; Hylden and Wilcox, 1980) at $20 \mathrm{ng} / 10 \mu \mathrm{l}$. AG490 (Sigma), an inhibitor of JAK2, was dissolved in ACSF at a concentration of $5 \mu \mathrm{M}$.

A solution of IL-6 $(10 \mu \mathrm{l})$ or ACSF $(10 \mu \mathrm{l})$ along with a further $10 \mu \mathrm{l}$ ACSF was injected via a micro syringe into the lumbar subarachnoid space of intact rats $(n=2$ for each group). Animals were left to survive for 1 day and cervical DRG (C6-C8) were removed following pericardial infusion with Zamboni fixative solution. Longitudinal DRG sections were double immunostained with mouse monoclonal antibody against GAP-43 (1:500; Sigma, USA) and rabbit polyclonal antiSTAT3 (Y705) antibody (1:100; Santa Cruz, CA, USA). The immunofluorescence reaction was visualized by treatment with FITC-conjugated donkey anti-mouse and TRITC-conjugated donkey anti-rabbit secondary antibodies (1:100; Millipore, USA) for $90 \mathrm{~min}$ at room temperature. Activation and nuclear translocation of STAT3 as well as GAP-43 immunofluorescence intensity were measured using a NIS-Elements image analysis system (Nikon, Czechia) as described previously.

To investigate in vivo the role of IL-6 in triggering the pro-regenerative state in cervical DRG, the right ulnar nerve was crushed as described $(n=8)$. A solution of IL-6 $(10 \mu \mathrm{l})$ or ACSF $(10 \mu \mathrm{l})$ along with a further $10 \mu \mathrm{l}$ ACSF was then injected via a micro syringe into the lumbar subarachnoid space $(n=4$ for each group). The ulnar nerves were removed 1 day later (after the crush and intrathecal administration of IL-6) and fixed with Zamboni fixative solution. Axon regeneration was assessed on longitudinal cryostat sections (10 $\mu \mathrm{m}$ thick) immunostained for SCG-10 and analyzed as described.

To test whether the JAK2/STAT3 signaling pathway is involved in inducing the pro-regenerative state of cervical DRG neurons after prior sciatic nerve injury, the right sciatic nerve of 8 rats was cut (CSNT). After 7 days, the rats with prior CSNT were re-operated to expose and crush the right ulnar nerve. $10 \mu \mathrm{l}$ of AG490 solution $(5 \mu \mathrm{M})$ or ACSF $(10 \mu \mathrm{l})$, along with a further $10 \mu \mathrm{l}$ ACSF was then injected via a micro syringe into the subarachnoid space of the cisterna magna ( $n=4$ for each group). The length of regenerated SCG10+ axons was assessed on longitudinal cryostat sections (10 $\mu \mathrm{m}$ thick) 1 day after the ulnar nerve crush and intrathecal injection of ACSF or AG490 as described above. In addition, cryostat sections of cervical DRG (C6-C8) ipsilateral to the ulnar nerve crush were double immunostained for GAP-43 and STAT3 and analyzed using a NIS-Elements image analysis system (Nikon, Czechia) as described above.

\section{Enzyme-Linked Immunosorbent Assay (ELISA) of IL-6 in Rat Plasma}

Three naive rats and those operated on to create SNC or CSNT for 1,3 , and 7 days ( $n=3$ for each group), as well as sham-operated rats for $3(n=3)$ and $7(n=3)$ days were sacrificed by $\mathrm{CO}_{2}$ inhalation. Blood samples were obtained by intracardiac puncture and collected into tubes containing heparin and protease inhibitor cocktail (LaRoche, Switzerland). Plasma was separated by centrifugation $(2,500 \mathrm{~g}$ for $12 \mathrm{~min}$ ) and stored at $-60^{\circ} \mathrm{C}$ until analyzed. Total protein was measured by Nanodrop ND-1000 (Thermo Scientific) and the level of IL-6 protein was assessed using an ELISA kit with a sensitivity of $5 \mathrm{pg} / \mathrm{ml}$ (BioSource International, USA) according to the manufacturer's instructions. Measurement was carried out on a SUNRISE Basic microplate reader (Tecan, Salzburg, Austria) and the data were normalized as picograms of IL-6 protein to $100 \mu \mathrm{g}$ of total protein. IL-6 protein levels were compared to baseline values in plasma from naïve rats, which was arbitrarily set as one. Data were expressed as mean \pm SD.

\section{Statistical Analyses}

Statistical differences between data of immunofluorescence intensities, western blot analysis and RT-PCR of naïve DRG neurons and DRG neurons of sham-operated rats or rats with SNC and CSNT were tested using a Mann-Whitney U-test $(p<0.05)$. The same statistical analysis was used to compare IL-6 protein levels in plasma of naïve and sham-operated or SNC- and CSNT-operated rats. The mean number of neurites per neuron and total neurite length per neuron were compared between DRG neurons of sham- and SNC- or CSNT-operated rats using one-way ANOVA and Tukey's post hoc test to determine statistical significance. All statistical analyses were performed using STATISTICA 9.0 software (StatSoft, Inc., Tulsa, OK, USA).

\section{RESULTS}

\section{Immunohistochemical and Western Blot Analysis of GAP-43 and SCG-10 Proteins}

Western blot analysis of GAP-43 in cervical DRG of rats surviving 1, 3, 7, and 14 days after SNC showed the protein level peaked at 7 days with a drop at 14 days when compared to cervical DRG of naïve or sham-operated rats (Figure 1). Therefore, subsequent analyses illustrating the pro-regenerative state of cervical DRG neurons after sciatic nerve lesions were performed in rats 7 days after SNC or CSNT. 
The lumbar and cervical DRG neurons of naïve and sham-operated rats displayed a low basal level of GAP-43 immunostaining. GAP-43 immunofluorescence increased strongly in large and medium-sized neurons of both ipsilateral and contralateral lumbar DRG 7 days after SNC and CSNT. Some medium- and small-sized neurons displayed only weak GAP-43 immunopositivity. Sections of cervical DRG also demonstrated a similar pattern of bilaterally increased GAP-43 immunofluorescence (Figure 2).

Image analysis revealed a bilateral increase of mean GAP-43 immunofluorescence intensity in all size-classes of neurons in both lumbar and cervical DRG after unilateral SNC and CSNT in comparison with naïve and sham-operated animals. Sciatic nerve injury induced a greater increase in GAP-43 immunofluorescence intensity in large neurons than in medium- and small-sized neurons of both lumbar and cervical DRG. Further, a more significant increase in GAP-43 immunofluorescence was measured bilaterally in lumbar (3.3-2.6 times) than in cervical DRG neurons (2.5-1.9 times) compared to naïve or sham-operated rats. However, no significant differences were found between individual size-classes of neurons in the SNC and CSNT experimental groups (Figure $\mathbf{2 M}$ ).

Basal SCG-10 immunofluorescence was very low or absent in the lumbar and cervical DRG neurons of naïve rats. Most neurons of all sizes in lumbar and cervical DRG of both sides displayed significantly enhanced SCG-10 immunofluorescence intensity 7 days after SNC and CSNT compared to DRG
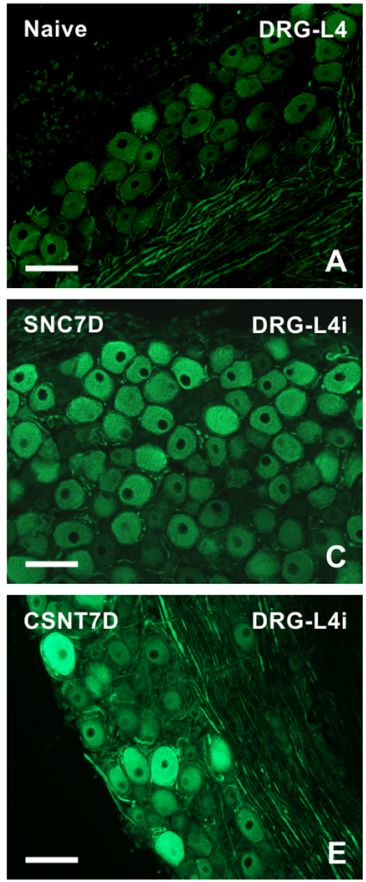

M
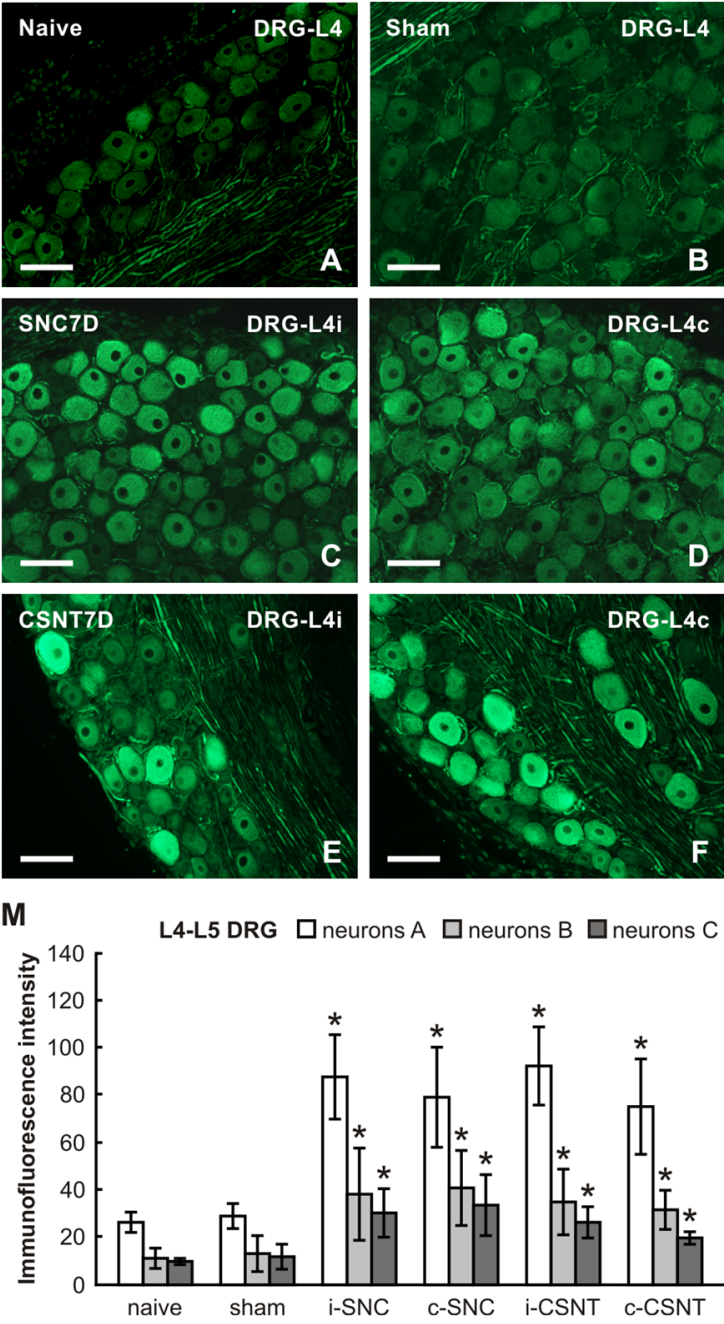

F
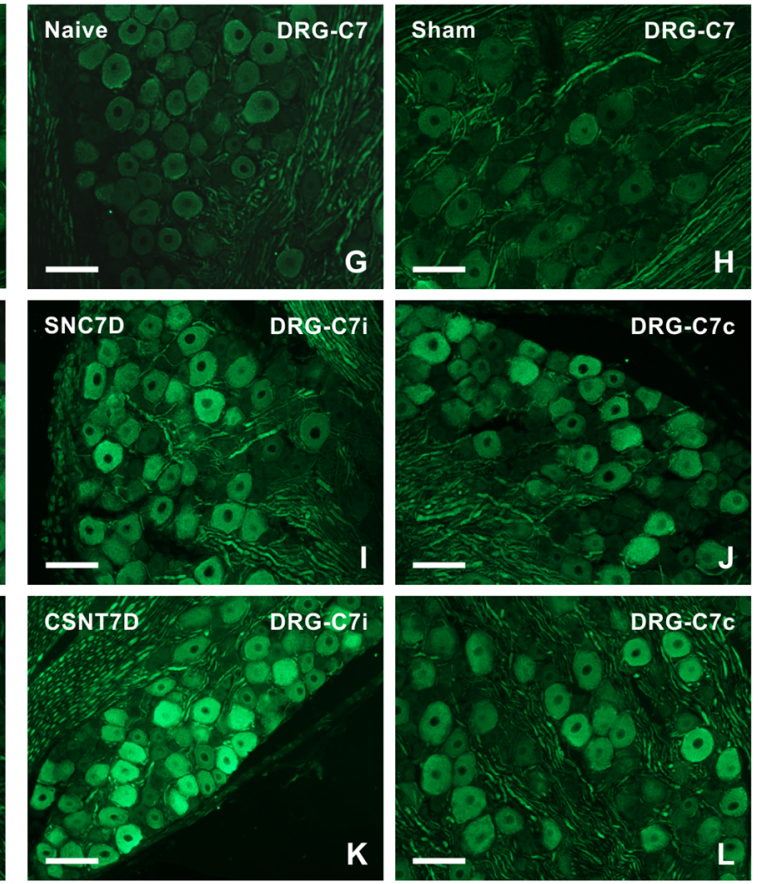

C6-C8 DRG $\square$ neurons A $\square$ neurons B $\square$ neurons C

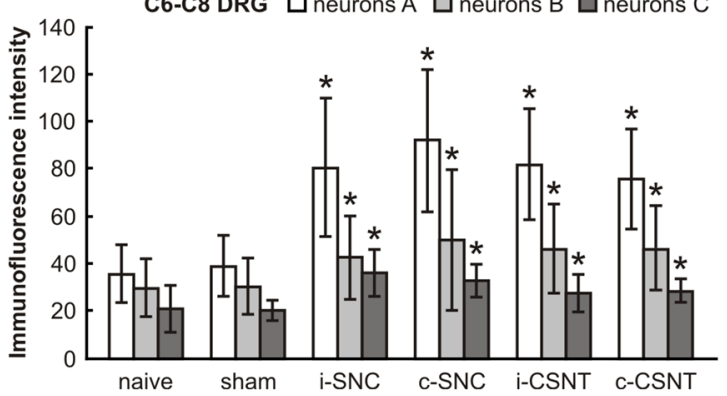

FIGURE 2 | Representative pictures of cryostat sections through the DRG from naiive (A,G), sham-operated (B,H) rats and rats with unilateral sciatic nerve compression (SNC; C,D,I,J) or complete sciatic nerve transection (CSNT; E,F,K,L) for 7 days. The sections of ipsilateral (C,E) and contralateral (D,F) DRG of the L4 spinal segment as well as ipsilateral $\mathbf{( I , K )}$ and contralateral $(\mathbf{J}, \mathbf{L})$ DRG of the $\mathrm{C7}$ spinal segment were incubated under the same conditions with mouse monoclonal antibody recognizing GAP-43. Scale bars $=75 \mu \mathrm{m}$. (M) Graph illustrating the mean intensity of GAP-43 immunofluorescence in individual DRG neuron-size classes of cervical (C6-C8) and lumbar (L4-L5) spinal segments ipsilateral (i) and contralateral (c) to unilateral SNC and transection (CSNT) for 7 days; $n=6$ for each group. Neurons A $\geq 40 \mu \mathrm{m}$; Neurons B 25-40 $\mu \mathrm{m}$; Neurons C $\leq 25 \mu \mathrm{m}$. *Significant difference $(p<0.05)$ compared to naive or sham-operated rats in a Mann-Whitney U-test. 

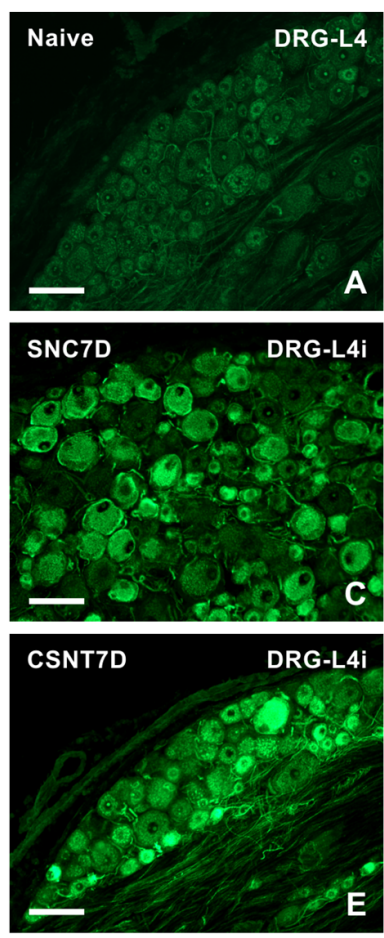

E

M
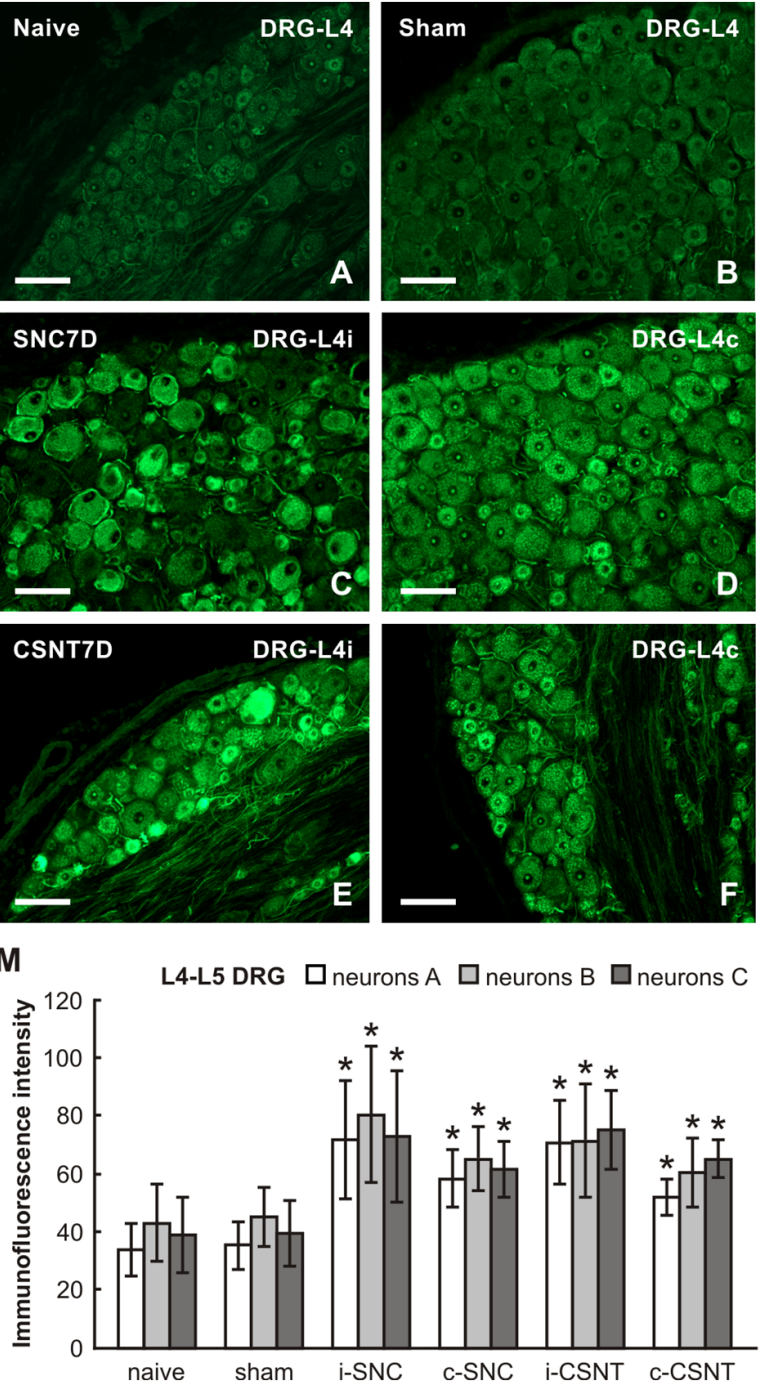

$F$
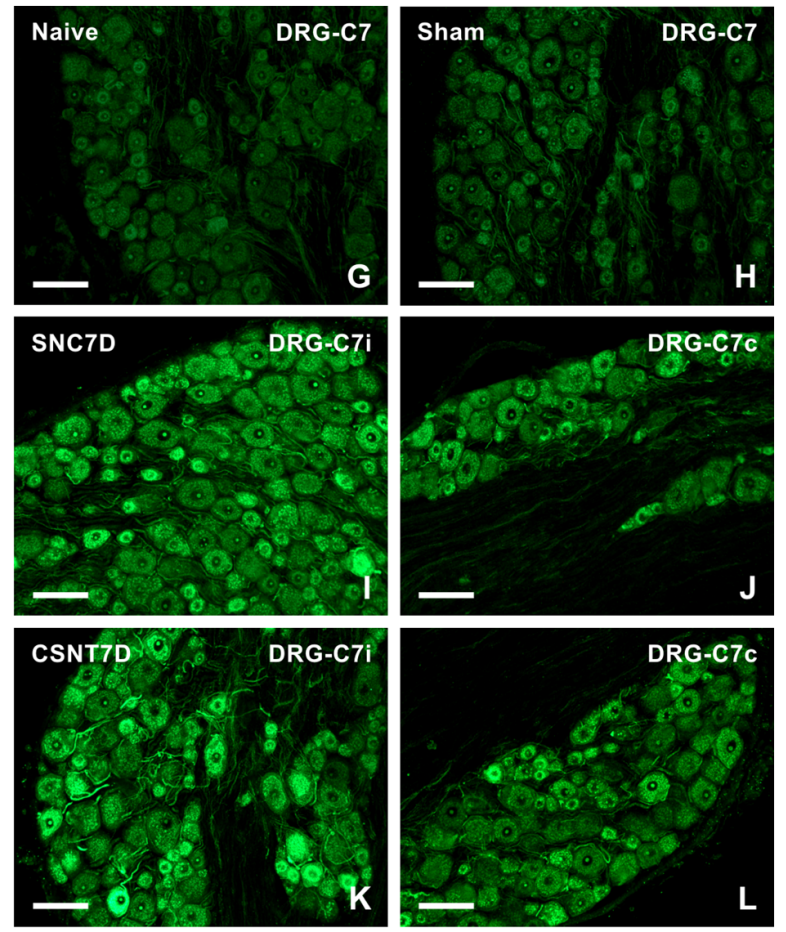

C6-C8 DRG $\square$ neurons A $\square$ neurons B $\square$ neurons C

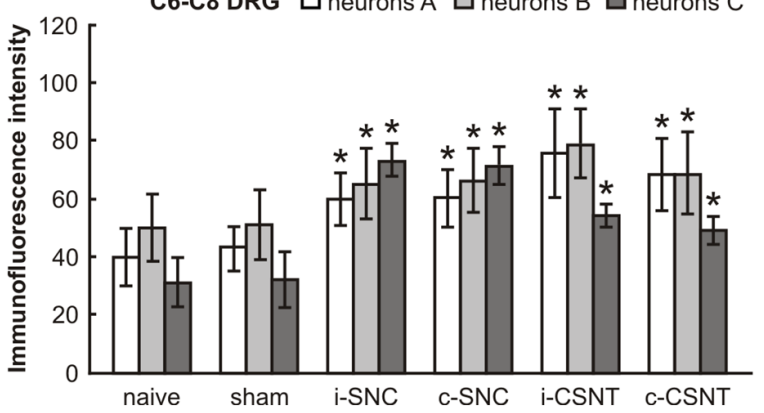

FIGURE 3 | Representative pictures of cryostat sections through the DRG from naive (A,G), sham-operated (B,H) rats and rats with unilateral SNC (C,D,I,J) or

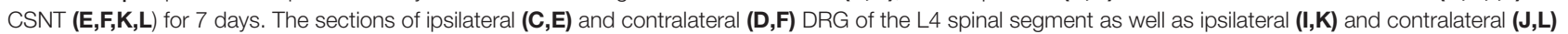
DRG of the C7 spinal segment were incubated under the same conditions with rabbit polyclonal antibody recognizing superior cervical ganglion-10 (SCG-10). Scale bars = $75 \mu \mathrm{m}$. (M) Graph illustrating the mean intensity of SCG-10 immunofluorescence measured in individual DRG neuron-size classes of cervical (C6-C8) and lumbar (L4-L5) spinal segments ipsilateral (i) and contralateral (c) to unilateral SNC and transection (CSNT) for 7 days; $n=6$ for each group. Neurons A $\geq 40 \mu \mathrm{m}$; Neurons B 25-40 $\mu \mathrm{m}$; Neurons $\mathrm{C} \leq 25 \mu \mathrm{m}$. *Significant difference $(p<0.05)$ compared to naive or sham-operated rats in a Mann-Whitney $U$-test.

from naïve or sham-operated rats. However, the increase in SCG-10 immunofluorescence intensity was lower than for GAP-43 (approximately 1.8-2 times in lumbar and 1.4-2.3 times in cervical DRG; Figures 3A-L). Moreover, image analysis of SCG-10 immunofluorescence intensity did not show any preferential increase among individual size-classes of DRG neurons (Figure 3M).

The increased levels of GAP-43 and SCG-10 proteins induced by SNC and CSNT and detected in the lumbar and cervical DRG of both sides by quantitative immunohistochemistry were verified by western blot analysis. Total GAP-43 and SCG-10 proteins were also significantly increased bilaterally in both lumbar and cervical DRG after unilateral SNC and CSNT for
7 days compared to naïve or sham-operated controls. Seven days after SNC or CSNT, the levels of GAP-43 protein were increased bilaterally in the lumbar and cervical DRG about twoto-three times compared to naïve and sham-operated controls. As was expected, GAP-43 protein levels shot up significantly in lumbar DRG after CSNT than SNC. Levels of GAP-43 protein in cervical DRG also increased significantly compared to controls, but the elevation was not to the extent seen in lumbar DRG (Figures 4A,B).

GAP-43 is activated by protein kinase C-mediated phosphorylation at serine 41 (S41) that promotes the polymerization and stabilization of filamentous actin (F-actin) related to axon growth and sprouting (Tsai et al., 2007). 


\section{A}

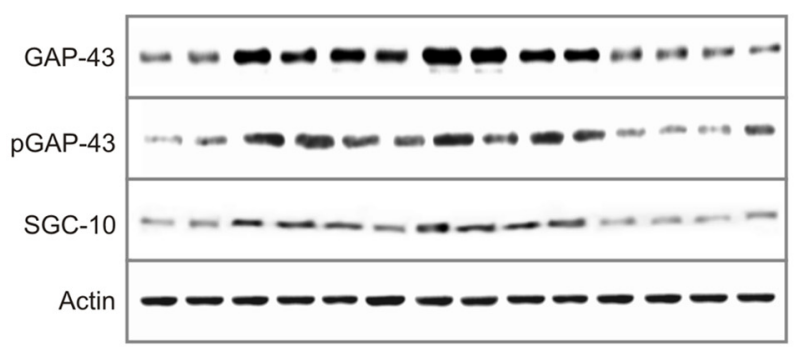

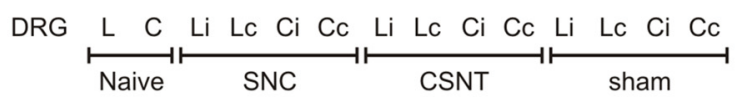

B
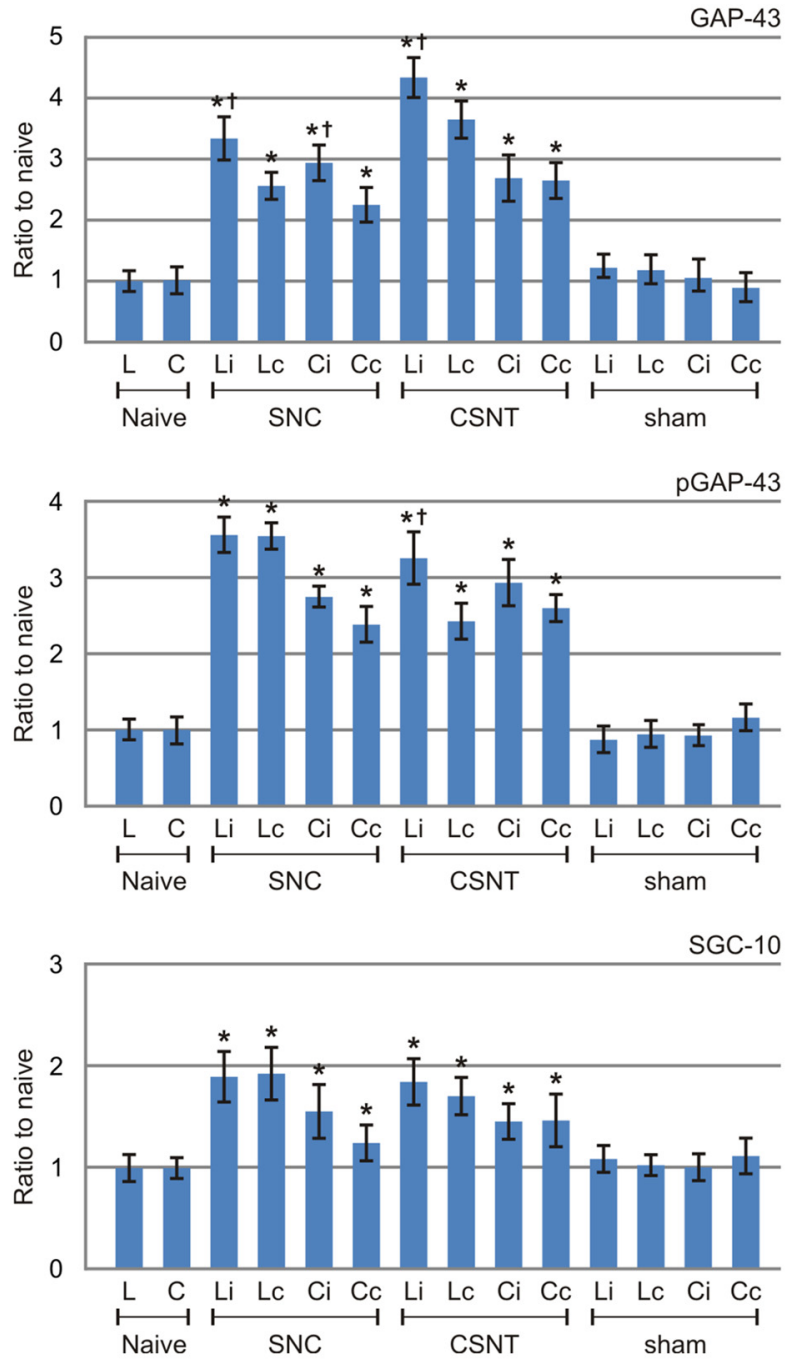

FIGURE 4 | Results of western blot analysis of GAP-43, pGAP-43 and SCG-10 protein levels in DRG of L4-L5 (L) and C6-C8 (C) segments removed from ipsilateral (i) and contralateral (C) sides of naïve as well as sham-, SNCand CSNT-operated rats for 7 days. Upper panel (A) illustrates representative western blots of DRG from three rats for each group. Equal loading of proteins was confirmed by actin levels (Actin). The same Actin controls were used for

(Continued)
FIGURE 4 | Continued

analysis of GAP-43, pGAP-43 and SCG-10 protein levels in this set representative western blots. Lower panels (B) show densitometry of individual protein bands after normalization to actin from three independent experiments; the intensities of the bands from naive DRG were as arbitrarily set to $1 .{ }^{*}$ Significant difference $(p<0.05)$ when compared to sham-operated rats; ${ }^{\dagger}$ Significant difference $(p<0.05)$ compared to contralateral DRG in a Mann-Whitney U-test.

Although in our experiments we used an antibody recognizing both unphosphorylated and phosphorylated GAP-43, the western blot analysis verified levels of activated GAP-43 using a specific antibody against phosphorylated GAP-43 (pGAP-43). The results demonstrated that activated pGAP-43 was increased bilaterally not only in lumbar but also in cervical DRG after SNC or CSNT compared to naïve or sham-operated controls. The magnitude of pGAP-43 increase was very similar to the increased GAP-43 levels. However, in contrast to significantly higher GAP-43 protein levels in ipsilateral than contralateral lumbar DRG after both SNC and CSNT, pGAP-43 protein level was significantly higher only in ipsilateral lumbar DRG after CSNT (Figures 4A,B).

In contrast to GAP-43 and pGAP-43, the increase in levels of SCG-10 was less marked (about 1.5-1.8-fold) and the increase was more or less the same on both sides. Moreover, no significant differences in SCG-10 levels were found in lumbar DRG from SNC- and CSNT-operated animals. Similar protein levels of SCG-10 were also measured in cervical DRG after SNC and CSNT, as in the case of GAP-43 and pGAP-43 (Figures 4A,B).

\section{RT-PCR Analysis of GAP-43 and SCG-10 mRNA}

RT PCR was used to determine the levels of the relevant GAP-43 and SCG-10 mRNA in cervical and lumbar DRG 7 days after unilateral sciatic nerve injury by SNC or CSNT. Samples of cervical and lumbar DRG from sham-operated animals displayed no significant changes in SCG-10 and GAP-43 mRNA levels compared to naïve controls.

Levels of GAP-43 and SCG-10 mRNAs were significantly increased bilaterally in both cervical and lumbar DRG 1 week following sciatic nerve injury by SNC or CSNT compared to naïve or sham-operated controls. GAP-43 mRNA increased bilaterally in lumbar DRG to a higher level after CSNT than SNC. However, a statistically significant difference between ipsilateral and contralateral lumbar DRG was found only in the CSNT group. In contrast to lumbar DRG, cervical ones displayed a bilateral increase of GAP-43 mRNA that was greater after SNC than after CSNT.

Similar to the GAP-43 and SCG-10 protein results, RT-PCR revealed a substantially smaller increase in SCG-10 mRNA levels in lumbar DRG (only up to 2.6-fold of sham-operated controls) than was measured for GAP-43 mRNA. Levels of SCG-10 mRNA were higher bilaterally in lumbar DRG after CSNT than after SNC, but the differences were not statistically significant. Both SNC and CSNT induced an approximately similar elevation of SCG-10 mRNA in cervical DRG of 


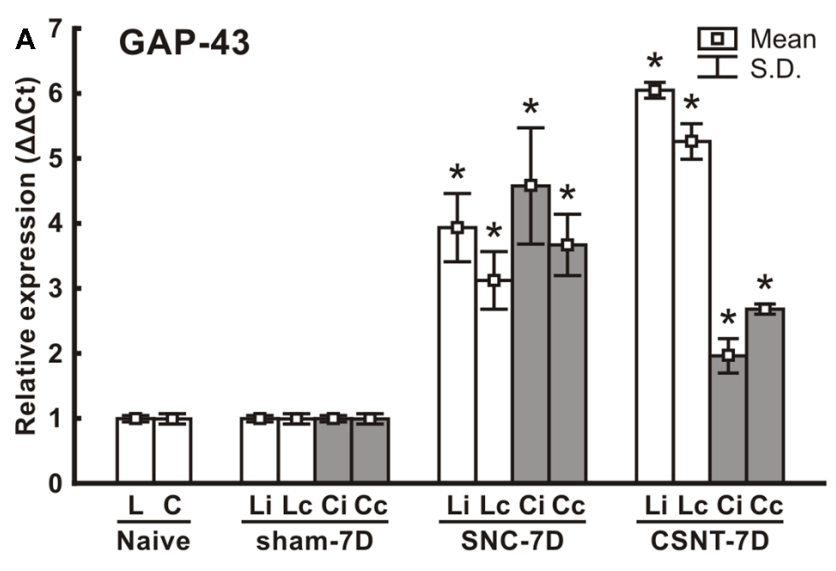

B

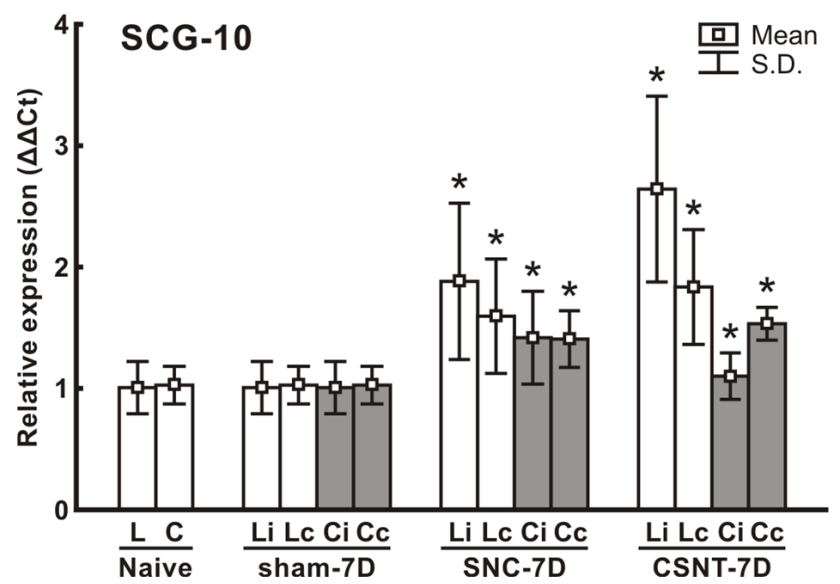

FIGURE 5 | Results of real-time PCR (RT-PCR) of relative GAP-43 (A) and SCG-10 (B) mRNA levels in DRG of lumbar (L4-L5) and cervical (C6-C8) spinal segments of removed from ipsilateral (i) and contralateral (c) sides of Naiive as well as sham-, SNC- and CSNT-operated rats for 7 days; $n=6$ for each group. Relative expressions were determined using Actin as the housekeeping gene and normalized to naïve controls. *Significant difference $(p<0.05)$ compared to sham-operated rats in a Mann-Whitney U-test.

both sides compared to naïve and sham-operated controls (Figure 5).

\section{Sciatic Nerve Lesion Induced Activation of cJun and p38 MAPK in Cervical DRG Neurons}

To confirm the sciatic nerve injury-induced pro-regenerative state of cervical DRG neurons demonstrated by GAP-43 and SCG10 analyses, activation of p-cJun and p-p38 MAPK was also investigated. Basal p-cJun and p-p38 MAPK immunofluorescence was very low in cervical DRG neurons of naïve and sham-operated rats. Sciatic nerve lesion increased immunofluorescence intensity of both p-cJun and p-p38 MAPK in the nuclei of cervical DRG neurons. Further, a higher intensity of p-p38 MAPK immunofluorescence was seen in the soma of neurons (Figure 6).

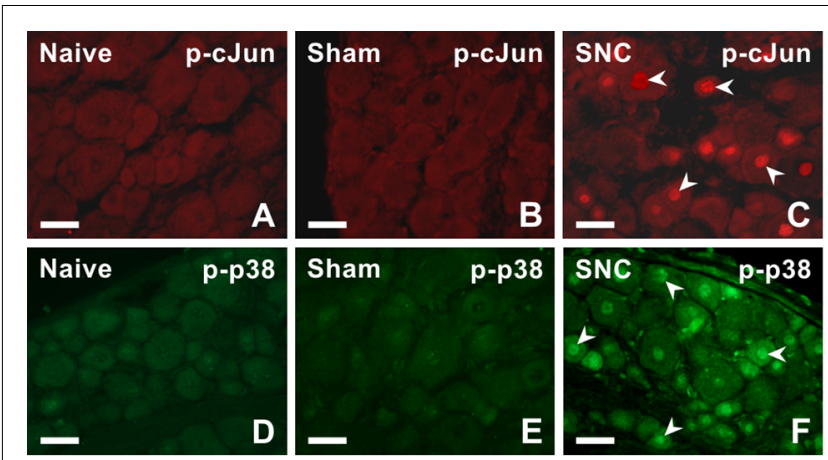

G

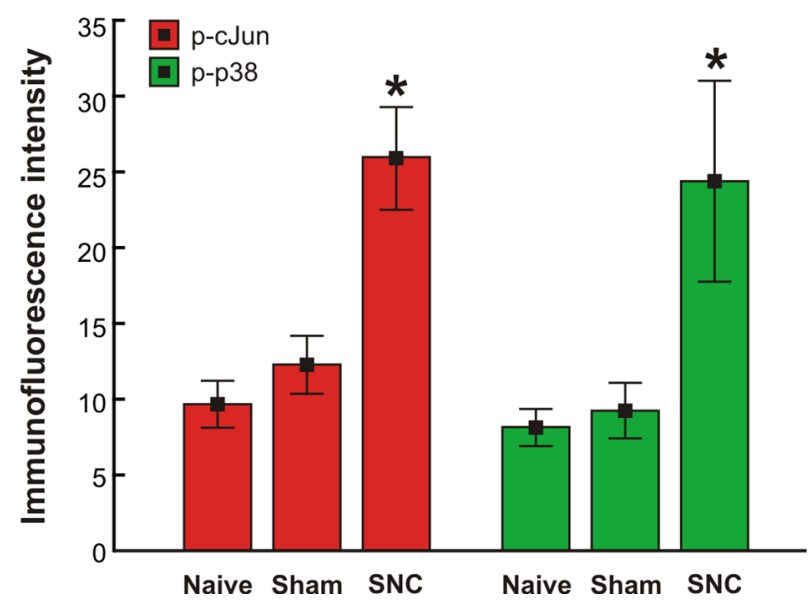

FIGURE 6 | Representative pictures of cervical (C7) DRG neurons of intact rats (A-D) and rats 7 day after sham- (B,E) or SNC-operation (C,F). DRG sections were immunostained for p-cJun (A-C) and p-p38 (D-F). Scale bars $=40 \mu \mathrm{m}$. (G) Graph illustrating mean immunofluorescence intensity of activated p-cJun (red columns) and p-p38 (green columns) in cervical DRG neurons of naïve, sham, and SNC groups. SNC induced activation of p-cJun and p-p38 (arrowheads, $\mathbf{C , G}$ ). *Significant difference $(p<0.05)$ compared to naïve or sham-operated rats in a Mann-Whitney U-test.

\section{Axon Regeneration Assay in Crushed Ulnar Nerve After Prior Sciatic Nerve Injury}

The axonal regeneration capacity of cervical DRG neurons was investigated in longitudinal sections of one-day-old crushed ulnar nerves by immunostaining for SCG-10-a specific marker for regenerated axons of primary sensory neurons (Shin et al., 2014). Axon regeneration index expressed as SCG-10+ axon extension from the point of nerve crush was greater in SNC- and CSNT-operated rats compared to control rats with only ulnar nerve crush (Figures 7A,B).

\section{In vitro Assay of Axonal Outgrowth Capacity of Cervical DRG Neurons After Sciatic Nerve Injury}

The increased capacity of cervical DRG neurons to regenerate their axons after prior sciatic nerve injury was verified by an in vitro assay of neurite outgrowth in DRG neurons taken from sham-, SNC- and CSNT-operated rats (Figures 8A-F). The diameter of neuronal bodies cultivated in vitro was between 


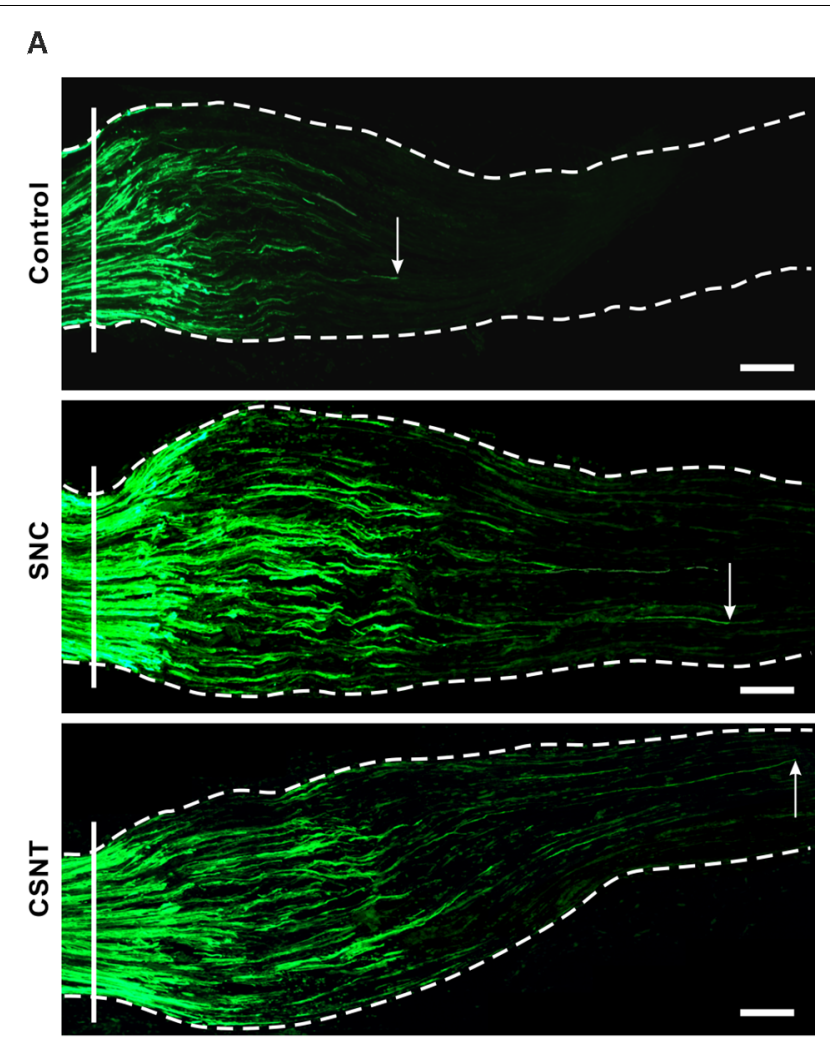

B

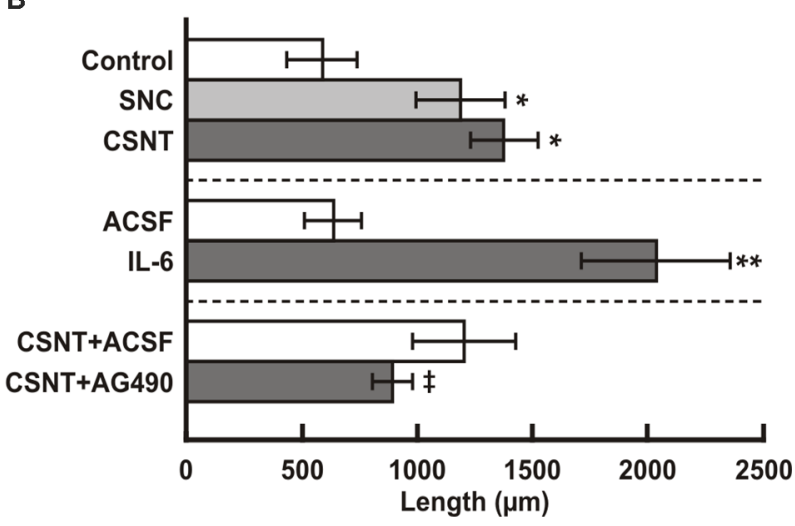

FIGURE 7 | (A) Representative longitudinal sections through the ulnar nerves distal to the crush site (solid line) showing SCG-10 positive regenerated axons. Arrows indicate the tip of the longest SCG-10 positive axons. The ulnar nerves were removed after 1 day from control rat (only ulnar nerve crush) and from rats 7 days after prior SNC or CSNT. Scale bars = $100 \mu \mathrm{m}$. (B) The top portion of the graph illustrates mean length of regenerated SCG10+ axons \pm SD in the ulnar nerve 1 day after crush in rats without a sciatic nerve injury (Control) and following 7 days from SNC or CSNT; $n=6$ for each group. *Significant difference $(p<0.05)$ compared to control. The middle portion illustrates mean length of regenerated SCG10+ axons $\pm S D$ in the ulnar nerve 1 day after crush and intrathecal injection of $10 \mu \mathrm{l}$ of artificial cerebrospinal fluid (ACSF) or IL-6 (20 ng/10 $\mu \mathrm{l}) ; n=4$ for each group. **Significant difference $(p<0.05)$ compared to control. The bottom portion illustrates mean length of regenerated SCG10+ axons \pm SD in the ulnar nerve 1 day after crush 7 days from prior CSNT and intrathecal application of ACSF (CSNT+ACSF) or JAK2 inhibitor (CSNT+AG490); $n=4$ for each group. ${ }^{\ddagger}$ Significant difference $(p<0.05)$ compared to CSNT+ACSF group in a Mann-Whitney U-test.
25 and $45 \mu \mathrm{m}$, thus encompassing all morphological classes of DRG neurons.

The mean number of neurites per neuronal body of cervical and lumbar DRG taken from sham-operated rats was very similar. Compared to cervical DRG neurons of sham-operated rats, the number of neurites sent off by cervical DRG neurons taken from SNC- and CSNT-operated rats was significantly higher. Surprisingly, cervical and contralateral lumbar DRG neurons displayed higher neurite outgrowth than lumbar DRG neurons ipsilateral to CSNT $(7.0 \pm 2.1$ and $7.0 \pm 1.7$ compared to $4.6 \pm 1.5$; Figure $8 \mathrm{G}$ ).

The total length of neurites per neuron was significantly larger in neurons from lumbar DRG of both sides and those cultivated from cervical DRG 7 days after SNC. Seven days after CSNT, the total neurite length per neuron was significantly larger in neurons cultivated from cervical and contralateral lumbar DRG, but not in neurons cultivated from ipsilateral lumbar DRG (Figure $\mathbf{8 H}$ ).

\section{IL-6 Protein Level in Plasma}

IL-6 protein levels increased significantly in the plasma of sham-operated rats at 3 days but returned to normal by 7 days after treatment. Plasma IL-6 levels were elevated also in SNC and CSNT rats at 1 and 3 days but dropped back close to normal 7 days after sciatic nerve lesions (Figure 9). These IL-6 plasma level measurements are consistent with our previous results following SNC (Dubový et al., 2013) and indicate that IL-6 in the blood is likely not inducing the pro-regenerative state in cervical DRG neurons 7 days after sciatic nerve lesion.

\section{Axon Regeneration Assay in Crushed Ulnar Nerve and Changes in the Pro-regenerative State in Cervical DRG Neurons After Intrathecal Application of IL-6 and JAK2 Inhibitor}

To investigate if IL-6 is responsible for activation of the pro-regenerative state of rat cervical DRG neurons, we intrathecally applied IL-6. We showed previously that intrathecal application of IL- 6 induced activation and nuclear translocation of STAT3 in DRG neurons (Dubový et al., 2018a). In the present experiments, we observed that intrathecal injection of IL-6 increased not only the activation of STAT3 but also the expression of GAP-43 in intact DRG neurons. In contrast, application of AG490 after CSNT and ulnar nerve crush resulted in decreased STAT3 activation and expression of GAP-43 in cervical DRG (Figure 10).

In addition, we measured the lengths of SCG-10+ regenerated axons distal to the ulnar nerve crush following intrathecal IL-6 application. The regenerated axons were significantly longer compared to control ulnar nerve crush or nerve crush and subsequent intrathecal injection of ACSF (Figure 7B, the middle portion). The pro-regenerative state of neurons is mediated by phosphorylation of STAT3 at the Y705 position by JAK2 (Schwaiger et al., 2000; Qiu et al., 2005; Niemi et al., 2016). When an inhibitor of JAK2 (AG490) was applied intrathecally in rats with ulnar nerve crush for a day following prior CSNT, SCG-10+ regenerated axons were significantly shorter compared to those 

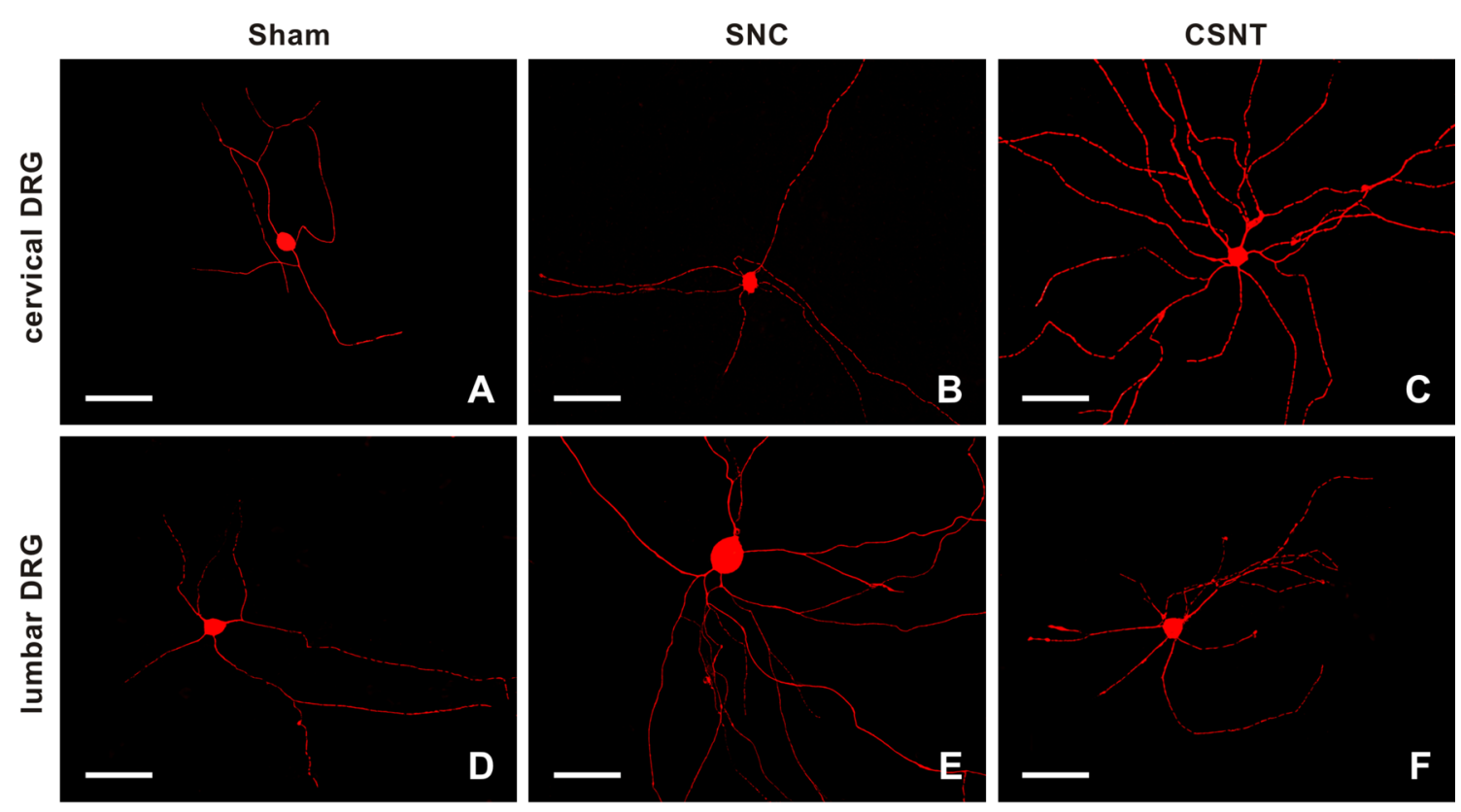

G

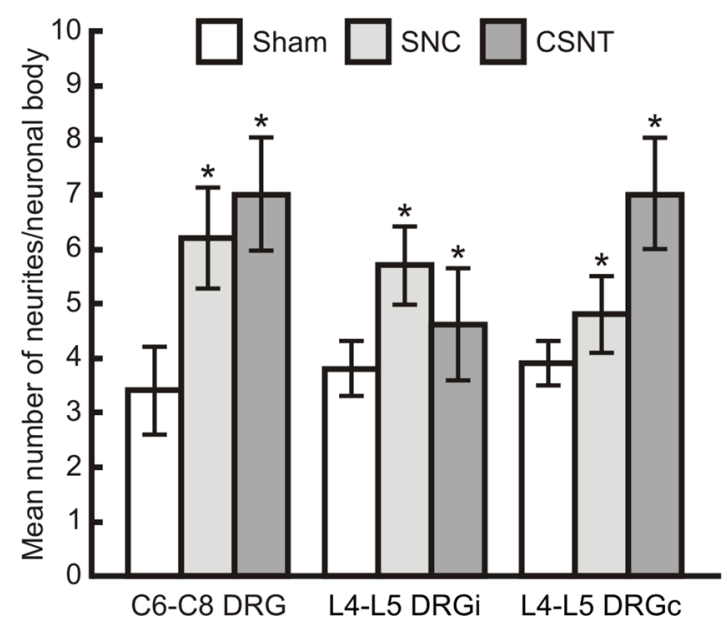

H

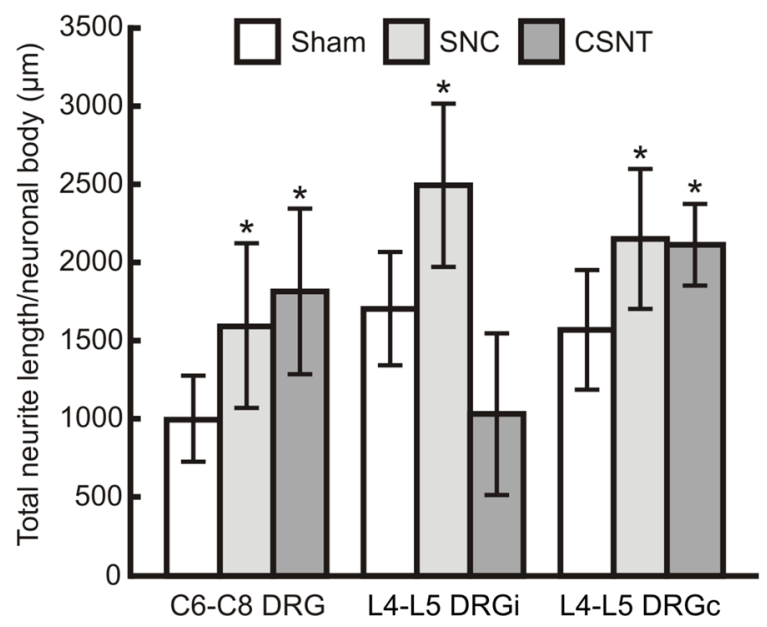

FIGURE 8 | (A-F) Representative pictures of cervical (C6-C8) and lumbar (L4-L5) DRG neurons of the ipsilateral side dissociated and cultured in vitro after removing of DRG from sham-, SNC- or CSNT-operated rats for 7 days. The DRG neurons were fixed and immunostained for $\beta$ III-tubulin after 2 days of in vitro incubation. Scale bars $=75 \mu \mathrm{m}$. The graphs illustrate mean number of neurites (G) and total neurite length $\mathbf{( H )}$ per neuronal body of in vitro cultivated DRG neurons of cervical (C6-C8) and lumbar (L4-L5) spinal segments from the ipsilateral (i) and contralateral (c) sides removed from sham-, SNC- and CSNT-operated rats ( $n=4$ for each group). At least 100 randomly selected neurons with nuclei per cover slip were measured. *Significant difference $(p<0.05)$ compared to sham-operated controls in a Mann-Whitney U-test.

of the rats subjected to CSNT and subsequent ulnar nerve crush and intrathecal injection only of ACSF (Figure 7B, the bottom portion).

\section{DISCUSSION}

Activation of the neuronal regenerative program after axon injury is an important prerequisite for useful reinnervation and functional recovery. This pro-regenerative status of the neurons is linked with the upregulation of regeneration-associated molecules (Ma and Willis, 2015). For example, regenerationassociated proteins like GAP-43 and SCG-10 are frequently used as molecular markers of the pro-regenerative state of neurons induced by experimental nerve injury. The DRG neurons are a useful model for studying the induction of the regenerationassociated program marked by increased levels of GAP-43 and SCG-10 mRNA and protein (Mason et al., 2002; Shin et al., 2014). The DRG neurons of naive rats display a low basal level of GAP-43 or SCG-10 immunostaining that is significantly increased in the neuronal bodies over a long period after sciatic 


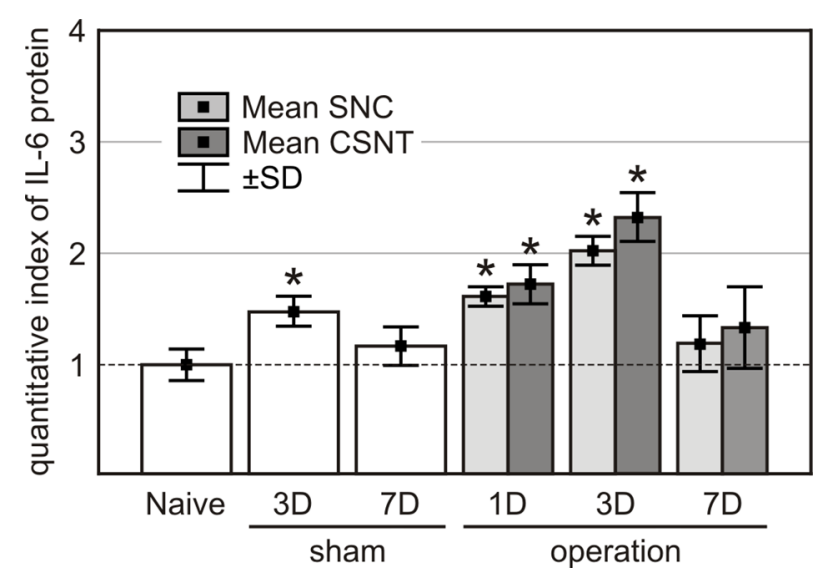

FIGURE 9 | |L-6 protein levels in the plasma of naïve, sham-, SNC- and CSNT-operated rats ( $n=3$ for each group). Blood samples of sham-operated rats were obtained 3 (3D) and 7 (7D) days after surgical treatment and those from SNC- and CSNT-operated rats were obtained 1 (1D), 3 (3D), and 7 (7D) days after operation. *Significant difference $(P<0.05)$ compared to baseline levels of naive rats in a Mann-Whitney $U$-test.

nerve injury (Stewart et al., 1992; Schreyer and Skene, 1993; Shin et al., 2014; Dubový et al., 2018b).

GAP-43 immunostaining is a widely used marker for regenerating axons in experimental models of peripheral nerve injury. Apart from regenerating axons, intense GAP-43 immunoreactivity is also present in Schwann and Schwannderived cells (Plantinga et al., 1993; Dubovy and Aldskogius, 1996). This GAP-43 immunostaining of Schwann cells associated with regenerated axons makes their detection difficult, especially during early periods of axon regeneration (Dubový et al., 2018b). In contrast to GAP-43, SCG-10 is transported from the soma to the proximal axonal stump very early after axotomy, whereas it is rapidly lost in distal axon stumps (Tararuk et al., 2006; Shin et al., 2012, 2014). In contrast to GAP-43, which labels regenerated axons after 3 days (Sar Shalom and Yaron, 2014), SCG-10 immunostaining is rapidly increased in proximal axonal stumps and regenerating sprouts within hours after axotomy (Shin et al., 2012, 2014). Moreover, SCG-10 is a marker for regenerated sensory axons of injured peripheral nerves (Shin et al., 2014). Therefore, we monitored GAP-43 and SCG-10 mRNA and protein to prove the pro-regenerative state of DRG neurons, and SCG-10 was used to assess axon regeneration distal to ulnar nerve crush after 1 day.

\section{Unilateral Sciatic Nerve Lesion Induced Pro-regenerative State in Both Lumbar and Cervical DRG}

Subpopulations of rat DRG neurons can be classified according their size, their cytological, chemical and physiological properties (Lawson, 2002) and behave differently with respect to their regeneration capacity as well as the expression of molecular regeneration markers (Bonilla et al., 2002; Leclere et al., 2007; Shin and Cho, 2017). Classically, DRG neurons are divided into large-, medium- and small-sized (Lawson et al., 1974)
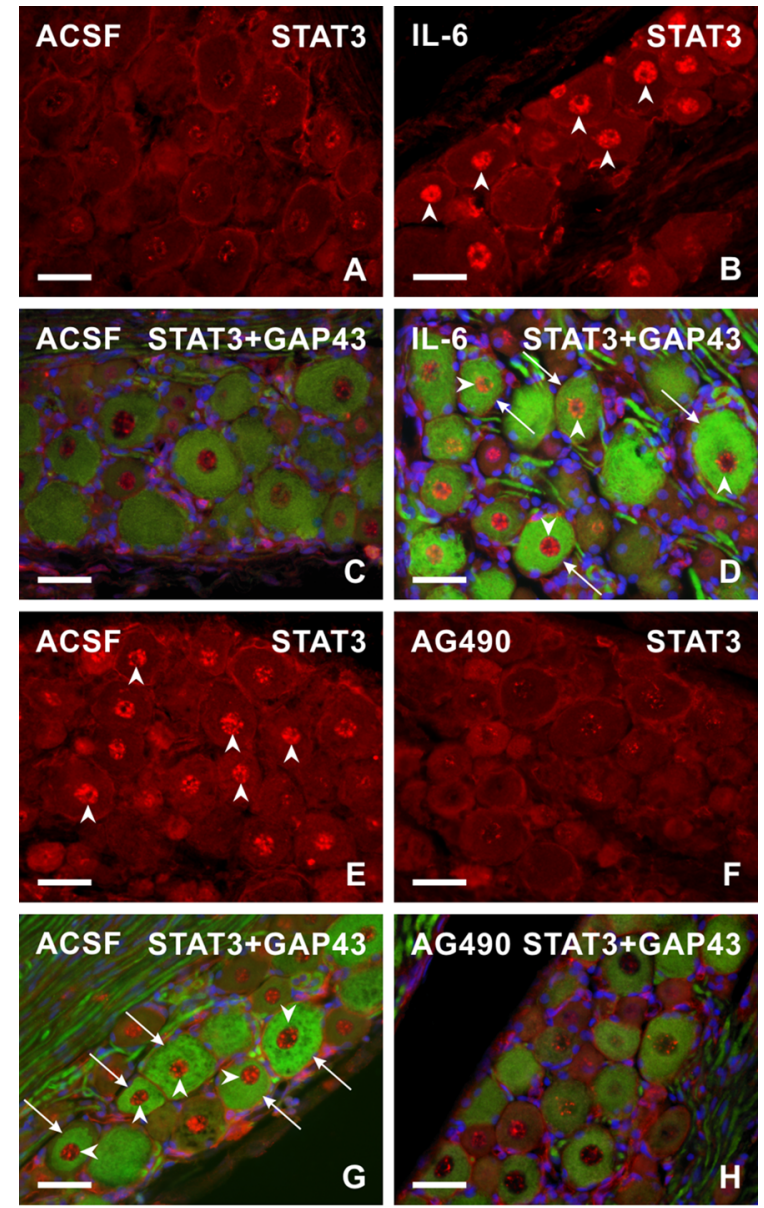

I

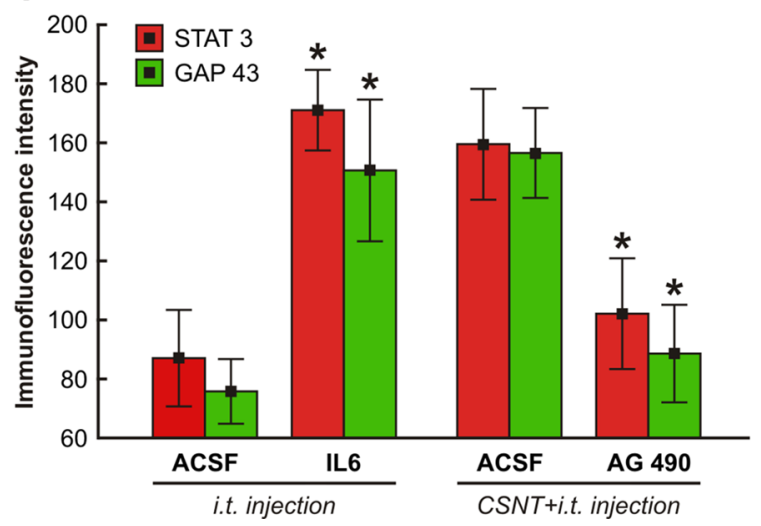

FIGURE 10 | Representative pictures of cervical (C7) DRG neurons of intact rats (A-D) and rats 1 day after the ulnar nerve crush following 7 days from CSNT (E-H). In intact rats, $10 \mu$ l of ACSF $(\mathbf{A}, \mathbf{C})$ or IL-6 (B,D) were intrathecally applied for 1 day. Cervical DRG were also removed from rats with CSNT for 7 days, a subsequent ulnar nerve crush for 1 day and intrathecal injection of $10 \mu$ l of ACSF (E,G) or AG490 (F,H). DRG sections were immunostained for STAT3 (A,B,E,F) or double immunostained (C,D, G,H) for STAT3 (red fluorescence) and GAP-43 (green fluorescence). Intrathecal injection of IL-6 induced activation and nuclear translocation of STAT3 (arrowheads, B,D) as well as increased immunostaining for GAP-43 in neurons (arrows, D) when compared with ACSF treatment (C). Cervical DRG (Continued) 


\section{FIGURE 10 | Continued}

neurons of rats following the ulnar nerve crush 7 days after CSNT displayed activation and nuclear translocation of STAT3 (E,G) and intense GAP-43 immunofluorescence in the neurons when injected with ACSF (G, arrowheads and arrows, respectively) while intrathecal application of AG490 resulted in a marked reduction of STAT3 activation and GAP-43 immunostaining (F,H). Scale bars $=40 \mu \mathrm{m}$. (I) Graph illustrating mean immunofluorescence intensity of activated STAT3 and GAP-43 in cervical DRG neurons of intact rats following intrathecal application of ACSF or IL-6 for 1 day and rats with CSNT for 7 days and intrathecal injection of ACSF or AG490. *Significant difference $(P<0.05)$ compared with ACSF-treated rats in a Mann-Whitney U-test.

and may also be identified by further molecular phenotyping. Quantitative immunohistochemical staining revealed increased GAP-43 and SCG-10 immunofluorescence in both lumbar and cervical DRG neurons ipsilateral as well as contralateral to the SNC or CSNT. Detailed image analysis showed that the predominant increase of GAP-43 immunofluorescence intensity was seen in the large-sized neuronal subpopulation of both lumbar and cervical DRG. In contrast, a similar elevation of SCG-10 immunofluorescence intensity was observed in all size-classes neurons of DRG in the lumbar and cervical spinal cord segments. Principally, bilateral increase of GAP-43 and SCG-10 proteins detected by quantitative immunohistochemical staining in both lumbar and cervical DRG neurons after sciatic nerve lesion was verified by measuring whole GAP-43 and SCG-10 protein levels using western blot analysis. Further, it also revealed that unilateral sciatic nerve lesion induced bilateral elevation of activated (phosphorylated) GAP-43 in both lumbar and cervical DRG.

The pro-regenerative state of DRG neurons in both lumbar and cervical spinal cord segments was confirmed by increased levels of GAP-43 and SCG-10 mRNA. Although SCG-10 is considered a better marker of the pro-regenerative state of DRG neurons (Shin et al., 2014; Dubový et al., 2018b), increases in the levels of GAP-43 mRNA and protein were more distinct compared to naïve or sham-operated rats in both lumbar and cervical DRG after SNC or CSNT for 7 days. Our observation of a bilateral increase in GAP-43 and SCG-10 mRNA and proteins correlates well with published results indicating that NGF mRNA is bilaterally increased in both cervical and lumbar DRG after unilateral sciatic nerve crush (Heumann et al., 1987; Wells et al., 1994). These results suggest that upregulation of growth-associated molecules is not restricted to axotomized DRG neurons and probably reflects a systemic response of DRG neurons to unilateral sciatic nerve injury (Dubový et al., 2013, 2018a).

The expression of regeneration-associated proteins like GAP-43 or SCG10 is operated by transcription factors, such as c-Jun (Raivich et al., 2004; Frey et al., 2015; Valakh et al., 2015) or members of the MAPK family, e.g., p38 (Verma et al., 2005; Temporin et al., 2008; Nix et al., 2011; Law et al., 2016). Our results showing increased levels of GAP-43 and SCG10 as well as activation of p-cJun and p-p38 in cervical DRG after sciatic nerve injury suggested that the pro-regenerative program can be induced in remote cervical DRG neurons 7 days after sciatic nerve lesion.

\section{Increased in vivo and in vitro Axon Regeneration Capacity of Cervical DRG Neurons Confirmed Their Pro-regenerative State Induced by Prior Sciatic Nerve Lesion}

While SCG-10 increases only in the proximal stumps of afferent axons (Shin et al., 2014), we also measured SCG-10 in regenerated peripheral arms of afferent axons associated with cervical DRG neurons distal to the ulnar nerve crush. SCG-10 immunopositive axons displayed a significantly greater length from the point of ulnar nerve crush after prior SNC or CSNT than in controls without the conditioning lesion. These results demonstrated in vivo the enhanced pro-regenerative state of cervical DRG neurons corresponding with increased levels of GAP-43 or SCG-10 induced by the conditioning sciatic nerve lesion.

Neurite outgrowth assays are used to assess the effects of in vivo manipulations performed prior to removing DRG from animals (Frey et al., 2015; Al-Ali et al., 2017). In our in vitro assay, the number of neurites and their total lengths per neuron were significantly higher in cervical DRG neurons removed from rats subjected to SNC or CSNT than those from sham-operated controls. The results of the in vitro assay confirmed the enhanced capacity of cervical DRG neurons to regenerate their neurites after the conditioning sciatic nerve lesion.

Thus, the axon outgrowth tested in vivo and in vitro proves the initiation of pro-regenerative state in cervical DRG neurons non-associated with sciatic nerve lesion. Our present results extend previously published results that unilateral nerve injury affects the uninjured contralateral nerve with respect to expression of inflammatory mediators (Ruohonen et al., 2002) including in vivo promotion of axonal regeneration in the contralateral nerve associated with enhanced cytokine expression in the contralateral DRG (Ryoke et al., 2000).

\section{IL-6 Is a Candidate for Signaling the Pro-regenerative Neuronal State in Remote DRG After Sciatic Nerve Lesion}

In response to unilateral sciatic nerve injury, mRNA and protein levels of IL- 6 and its receptors were enhanced bilaterally in primary sensory neurons not only in DRG of lumbar segments (L4-L5) associated with the injury, but also in cervical segments (C7-C8) not associated with the injured nerve (Brázda et al., 2013; Dubový et al., 2013). Upon binding to its membranebound receptor (IL-6R), IL-6 connects the intracellular regions of gp130 to initiate a signal transduction cascade by activating signal transducer and activator of transcription 3 (STAT3; Eulenfeld et al., 2012). Activation of STAT3 by JAK2-dependent phosphorylation at the tyrosine-705 (Y705) position occurs in DRG neurons after a nerve lesion and is mediated by neuropoietic cytokines including IL-6 (Schwaiger et al., 2000; Sheu et al., 2000; Qiu et al., 2005; Miao et al., 2006) and neurotrophins (Ng et al., 2006; Pellegrino and Habecker, 2013). We found STAT3 activation and nuclear translocation bilaterally in the DRG neurons of both lumbar and cervical spinal cord segments after unilateral SNC or CSNT. Moreover, 
we also proved increased levels of IL- 6 protein in the CSF following nerve injury as well as activation and nuclear translocation of STAT3 in DRG neurons along the neuroaxis after intrathecal injection of IL-6 (Dubový et al., 2018a). It is known that activation of STAT3 by JAK2 phosphorylation is a prerequisite for the synthesis of regeneration-associated proteins like GAP-43 or SCG-10 (Patodia and Raivich, 2012) and axon regeneration (Bareyre et al., 2011; Niemi et al., 2016). Intrathecal application of IL-6 in our present experiments increased STAT3 activation and GAP-43 expression in cervical DRG neurons, while a JAK2 inhibitor (AG490) decreased STAT3 activation and nuclear translocation as well as expression of GAP-43.

Intrathecal delivery of IL-6 promotes regeneration of the central arms of afferent axons into the spinal dorsal cord. This then activates the neuronal regeneration program in DRG neurons to overcome inhibitors of axon regeneration present in myelin (Cao et al., 2006). Therefore, in our experiments we tested in vivo the effect of intrathecal IL-6 and JAK2 inhibitor injection on axon regeneration after an ulnar nerve crush to illustrate the pro-regenerative state of cervical DRG neurons. Intrathecal IL-6 injection significantly increased the lengths of SCG-10+ regenerated axons distal to the ulnar nerve crush. In contrast, intrathecal application of AG490 reduced the lengths of regenerated axons after the ulnar nerve crush following prior unilateral SNC or CSNT. These results suggested a critical role for the JAK/STAT signaling pathway activated by IL-6 in inducing the pro-regenerative state in remote DRG neurons after unilateral sciatic nerve lesion.

What remains unclear is the mode of activation of remote DRG neurons following a conditioning unilateral sciatic nerve lesion. Axonal transport of local WD signaling molecules would be implicated only on the ipsilateral side of the compressed nerve (SNC) but can be excluded when the similar bilateral changes were found in CSNT group of rats. We observe bilateral changes in DRG of both lumbar and cervical segments, and this rather points towards a systemic pathway through either the blood stream or the CSF. The low level of IL-6 in the plasma of rats 7 days after SNC or CSNT suggests that it is more likely to be via the CSF. We found previously that rat DRG have no barrier to the CSF of the spinal subarachnoid space (Joukal et al., 2016). Therefore, intrathecal injection of IL-6 with subsequent activation of STAT3 in cervical DRG neurons (Dubový et al., 2018a) can trigger upregulation of GAP-43 and SCG-10 associated with the pro-regenerative state of these neurons. A unilateral sciatic nerve lesion induces increased IL-6 synthesis in the corresponding lumbar DRG (Murphy et al., 1995; Dubový et al., 2013) and the cytokine molecules can be released

\section{REFERENCES}

Abe, N., Borson, S. H., Gambello, M. J., Wang, F., and Cavalli, V. (2010). Mammalian target of rapamycin (mTOR) activation increases axonal growth capacity of injured peripheral nerves. J. Biol. Chem. 285, 28034-28043. doi: 10.1074/jbc.m110.125336

Al-Ali, H., Beckerman, S. R., Bixby, J. L., and Lemmon, V. P. (2017). In vitro models of axon regeneration. Exp. Neurol. 287, 423-434. doi: 10.1016/j. expneurol.2016.01.020 into the subarachnoid space and transported via CSF into the remote DRG to activate STAT3 (Dubový et al., 2018a) and this then induces the neuronal pro-regenerative state.

Finally, our results indicate that the pro-regenerative state of cervical DRG neurons illustrates a systemic reaction along the neuroaxis to unilateral sciatic nerve injury, and that this reaction can be mediated by IL-6 and JAK2/STAT3 signaling. Moreover, the results suggest a role for inflammatory mediators in activating the neuronal pro-regenerative state without direct retrograde axonal transport of signaling molecules from the injured nerve.

\section{CONCLUSION}

In the present study, we have demonstrated that sciatic nerve lesion for 7 days triggers bilateral activation of a pro-regenerative state not only in the primary sensory neurons of lumbar DRG (L4-L5) associated with injured nerve but also in remote cervical DRG. The bilateral activation of the pro-regenerative state in DRG neurons anatomically non-associated with the injured nerve correlates well with our previous observation of bilateral expression of IL-6 and activation of STAT3. Taken together, these results indicate that the systemic reaction of DRG neurons to a unilateral nerve lesion along the neuroaxis can be mediated by IL- 6 and JAK2/STAT3 signaling. This phenomenon can be activated also by other molecules released into the CSF of the perispinal subarachnoid space by neurons and non-neuronal cells in DRG associated with the injured nerve.

\section{AUTHOR CONTRIBUTIONS}

PD designed the research and wrote the article. IK, IH-S and MJ performed in vivo experiments and prepared samples for immunohistochemistry, western blot and RT-PCR and wrote the corresponding texts. MK performed in vitro experiments and prepared samples for immunohistochemistry and wrote the corresponding text. VB performed and analyzed western blot and RT-PCR data and wrote the corresponding text.

\section{FUNDING}

This work was supported by grant No. 16-08508S of The Czech Science Foundation (Grantová Agentura České Republiky).

\section{ACKNOWLEDGMENTS}

We thank Ms. Dana Kutějová, Ms. Marta Lněnícková, Ms. Jitka Mikulášková, Mgr. Jana Vachová and Mr. Lumír Trenčanský for their skillful technical assistance.

Bareyre, F. M., Garzorz, N., Lang, C., Misgeld, T., Buning, H., and Kerschensteiner, M. (2011). In vivo imaging reveals a phase-specific role of STAT3 during central and peripheral nervous system axon regeneration. Proc. Natl. Acad. Sci. U S A 108, 6282-6287. doi: 10.1073/pnas.10152 39108

Bonilla, I. E., Tanabe, K., and Strittmatter, S. M. (2002). Small proline-rich repeat protein $1 \mathrm{~A}$ is expressed by axotomized neurons and promotes axonal outgrowth. J. Neurosci. 22, 1303-1315. doi: 10.1523/jneurosci.22-04-01303. 2002 
Brázda, V., Klusáková, I., Svíženská, I. H., and Dubovy, P. (2013). Dynamic response to peripheral nerve injury detected by in situ hybridization of IL-6 and its receptor mRNAs in the dorsal root ganglia is not strictly correlated with signs of neuropathic pain. Mol. Pain 9:42. doi: 10.1186/1744-80 $69-9-42$

Brazda, V., Muller, P., Brozkova, K., and Vojtesek, B. (2006). Restoring wild-type conformation and DNA-binding activity of mutant p53 is insufficient for restoration of transcriptional activity. Biochem. Biophys. Res. Commun. 351, 499-506. doi: 10.1016/j.bbrc.2006.10.065

Cafferty, W. B. J., Gardiner, N. J., Gavazzi, I., Powell, J., McMahon, S. B., Heath, J. K., et al. (2001). Leukemia inhibitory factor determines the growth status of injured adult sensory neurons. J. Neurosci. 21, 7161-7170. doi: 10.1523/jneurosci.21-18-07161.2001

Cao, Z. X., Gao, Y., Bryson, J. B., Hou, J. W., Chaudhry, N., Siddiq, M., et al. (2006). The cytokine interleukin-6 is sufficient but not necessary to mimic the peripheral conditioning lesion effect on axonal growth. J. Neurosci. 26, 5565-5573. doi: 10.1523/jneurosci.0815-06.2006

Chandran, V., Coppola, G., Nawabi, H., Omura, T., Versano, R., Huebner, E. A., et al. (2016). A systems-level analysis of the peripheral nerve intrinsic axonal growth program. Neuron 89, 956-970. doi: 10.1016/j.neuron.2016.01.034

Christie, K. J., Webber, C. A., Martinez, J. A., Singh, B., and Zochodne, D. W. (2010). PTEN inhibition to facilitate intrinsic regenerative outgrowth of adult peripheral axons. J. Neurosci. 30, 9306-9315. doi: 10.1523/jneurosci.6271-09. 2010

Dubovy, P., and Aldskogius, H. (1996). Growth-associated protein (GAP-43) in terminal Schwann cells of rat Pacinian corpuscles. Neuroreport 7, 2147-2150. doi: 10.1097/00001756-199609020-00017

Dubový, P., Brázda, V., Klusáková, I., and Hradilová-Sviženská, I. (2013). Bilateral elevation of interleukin-6 protein and mRNA in both lumbar and cervical dorsal root ganglia following unilateral chronic compression injury of the sciatic nerve. J. Neuroinflammation 10:55. doi: 10.1186/1742-2094-10-55

Dubový, P., Hradilová-Svíženská, I., Klusáková, I., Kokošová, V., Brázda, V., and Joukal, M. (2018a). Bilateral activation of STAT3 by phosphorylation at the tyrosine-705 (Y705) and serine-727 (S727) positions and its nuclear translocation in primary sensory neurons following unilateral sciatic nerve injury. Histochem. Cell Biol. 150, 37-47. doi: 10.1007/s00418-018-1656-y

Dubový, P., Klusáková, I., Hradilová-Svíženská, I., and Joukal, M. (2018b). Expression of regeneration-associated proteins in primary sensory neurons and regenerating axons after nerve injury-An overview. Anat. Rec. 301, 1618-1627. doi: 10.1002/ar.23843

Dubový, P., Klusáková, I., and Svízenská, I. (2002). A quantitative immunohistochemical study of the endoneurium in the rat dorsal and ventral spinal roots. Histochem. Cell Biol. 117, 473-480. doi: 10.1007/s00418002-0411-5

Eulenfeld, R., Dittrich, A., Khouri, C., Muller, P. J., Mutze, B., Wolf, A., et al. (2012). Interleukin-6 signalling: More than Jaks and STATs. Eur. J. Cell Biol. 91, 486-495. doi: 10.1016/j.ejcb.2011.09.010

Frey, E., Valakh, V., Karney-Grobe, S., Shi, Y., Milbrandt, J., and DiAntonio, A. (2015). An in vitro assay to study induction of the regenerative state in sensory neurons. Exp. Neurol. 263, 350-363. doi: 10.1016/j.expneurol.2014.10.012

Heumann, R., Lindholm, D., Bandtlow, C., Meyer, M., Radeke, M. J., Misko, T. P., et al. (1987). Differential regulation of messenger-rna encoding nerve growthfactor and its receptor in rat sciatic-nerve during development, degeneration and regeneration - role of macrophages. Proc. Natl. Acad. Sci. U S A 84, 8735-8739. doi: 10.1073/pnas.84.23.8735

Hylden, J. L. K., and Wilcox, G. L. (1980). Intrathecal morphine in mice: a new technique. Eur. J. Pharmacol. 67, 313-316. doi: 10.1016/0014-2999(80)90515-4

Itoh, A., Horiuchi, M., Bannerman, P., Pleasure, D., and Itoh, T. (2009). Impaired regenerative response of primary sensory neurons in ZPK/DLK gene-trap mice. Biochem. Biophys. Res. Commun. 383, 258-262. doi: 10.1016/j.bbrc.2009. 04.009

Joukal, M., Klusáková, I., and Dubový, P. (2016). Direct communication of the spinal subarachnoid space with the rat dorsal root ganglia. Ann. Anat. 205, 9-15. doi: 10.1016/j.aanat.2016.01.004

Law, V., Dong, S., Rosaless, J. L., Jeong, M. Y., Zochodne, D., and Lee, K. Y. (2016). Enhancement of peripheral nerve regrowth by the purine nucleoside analog and cell cycle inhibitor, Roscovitine. Front. Cell. Neurosci. 10:238. doi: $10.3389 /$ fncel.2016.00238
Lawson, S. N. (2002). Phenotype and function of somatic primary afferent nociceptive neurones with C-, A $\delta$ - or A $\alpha / \beta$-fibres. Exp. Physiol. 87, 239-244. doi: $10.1113 /$ eph8702350

Lawson, S. N., Caddy, K. W. T., and Biscoe, T. J. (1974). Development of rat dorsal root ganglion neurons. Studies of cell birthdays and changes in mean cell diameter. Cell Tissue Res. 153, 399-413. doi: 10.1007/bf00229167

Leclere, P. G., Norman, E., Groutsi, F., Coffin, R., Mayer, U., Pizzey, J., et al. (2007). Impaired axonal regeneration by isolectin B4-binding dorsal root ganglion neurons in vitro. J. Neurosci. 27, 1190-1199. doi: 10.1523/jneurosci.5089-06. 2007

Liabotis, S., and Schreyer, D. J. (1995). Magnitude of GAP-43 induction following peripheral axotomy of adult-rat dorsal-root ganglion neurons is independent of lesion distance. Exp. Neurol. 135, 28-35. doi: 10.1006/exnr.1995.1063

Liu, K., Tedeschi, A., Park, K. K., and He, Z. (2011). Neuronal intrinsic mechanisms of axon regeneration. Ann. Rev. Neurosci. 34, 131-152. doi: 10.1146/annurev-neuro-061010-113723

Ma, T. C., and Willis, D. E. (2015). What makes a RAG regeneration associated? Front. Mol. Neurosci. 8:43. doi: 10.3389/fnmol.2015.00043

Mar, F. M., Bonni, A., and Sousa, M. M. (2014). Cell intrinsic control of axon regeneration. EMBO Rep. 15, 254-263. doi: 10.1002/embr.2013 37723

Mason, M. R. J., Lieberman, A. R., Grenningloh, G., and Anderson, P. N. (2002). Transcriptional upregulation of SCG10 and CAP-23 is correlated with regeneration of the axons of peripheral and central neurons in vivo. Mol. Cell. Neurosci. 20, 595-615. doi: 10.1006/mcne.2002.1140

Miao, T., Wu, D. S., Zhang, Y., Bo, X. N., Subang, M. C., Wang, P., et al. (2006). Suppressor of cytokine signaling-3 suppresses the ability of activated signal transducer and activator of transcription-3 to stimulate neurite growth in rat primary sensory neurons. J. Neurosci. 26, 9512-9519. doi: 10.1523/jneurosci. 2160-06.2006

Murphy, P. G., Grondin, J., Altares, M., and Richardson, P. M. (1995). Induction of interleukin-6 in axotomized sensory neurons. J. Neurosci. 15, 5130-5138. doi: 10.1523/jneurosci.15-07-05130.1995

Neumann, S., and Woolf, C. J. (1999). Regeneration of dorsal column fibers into and beyond the lesion site following adult spinal cord injury. Neuron 23, 83-91. doi: 10.1016/s0896-6273(00)80755-2

Ng, Y. P., Cheung, Z. H., and Ip, N. Y. (2006). STAT3 as a downstream mediator of Trk signaling and functions. J. Biol. Chem. 281, 15636-15644. doi: 10.1074/jbc. m601863200

Niemi, J. P., DeFrancesco-Lisowitz, A., Cregg, J. M., Howarth, M., and Zigmond, R. E. (2016). Overexpression of the monocyte chemokine CCL2 in dorsal root ganglion neurons causes a conditioning-like increase in neurite outgrowth and does so via a STAT3 dependent mechanism. Exp. Neurol. 275, 25-37. doi: 10.1016/j.expneurol.2015.09.018

Nix, P., Hisamoto, N., Matsumoto, K., and Bastiani, M. (2011). Axon regeneration requires coordinate activation of p38 and JNK MAPK pathways. Proc. Natl. Acad. Sci. U S A 108, 10738-10743. doi: 10.1073/pnas.1104830108

Patodia, S., and Raivich, G. (2012). Role of transcription factors in peripheral nerve regeneration. Cell Tissue Res. 5:8. doi: 10.3389/fnmol.2012.00008

Pellegrino, M. J., and Habecker, B. A. (2013). STAT3 integrates cytokine and neurotrophin signals to promote sympathetic axon regeneration. Mol. Cell. Neurosci. 56, 272-282. doi: 10.1016/j.mcn.2013.06.005

Plantinga, L. C., Verhaagen, J., Edwards, P. M., Hol, E. M., Bär, P. R., and Gispen, W. H. (1993). The expression of B-50/GAP-43 in Schwann-cells is up-regulated in degenerating peripheral-nerve stumps following nerve injury. Brain Res. 602, 69-76. doi: 10.1016/0006-8993(93)90243-g

Pool, M., Thiemann, J., Bar-Or, A., and Fournier, A. E. (2008). NeuriteTracer: a novel ImageJ plugin for automated quantification of neurite outgrowth. J. Neurosci. Methods 168, 134-139. doi: 10.1016/j.jneumeth.2007.08.029

Qiu, J., Cafferty, W. B. J., McMahon, S. B., and Thompson, S. W. N. (2005). Conditioning injury-induced spinal axon regeneration requires signal transducer and activator of transcription 3 activation. J. Neurosci. 25, 1645-1653. doi: 10.1523/JNEUROSCI.3269-04.2005

Raivich, G., Bohatschek, M., Da Costa, C., Iwata, O., Galiano, M., Hristova, M., et al. (2004). The AP-1 transcription factor c-jun is required for efficient axonal regeneration. Neuron 43, 57-67. doi: 10.1016/j.neuron.2004.06.005

Rishal, I., and Fainzilber, M. (2014). Axon-soma communication in neuronal injury. Nat. Rev. Neurosci. 15, 32-42. doi: 10.1038/nrn3609 
Ronchi, G., Nicolino, S., Raimondo, S., Tos, P., Battiston, B., Papalia, B., et al. (2009). Functional and morphological assessment of a standardized crush injury of the rat median nerve. J. Neurosci. Methods 179, 51-57. doi: 10.1016/j. jneumeth.2009.01.011

Ruohonen, S., Jagodi, M., Khademi, M., Taskinen, H. S., Ojala, P., Olsson, T., et al. (2002). Contralateral non-operated nerve to transected rat sciatic nerve shows increased expression of IL-1 $\beta$, TGF- $\beta$ 1, TNF- $\alpha$, and IL-10. J. Neuroimmunol. 132, 11-17. doi: 10.1016/s0165-5728(02)00281-3

Ryoke, K., Ochi, M., Iwata, A., Uchio, Y., Yamamoto, S., and Yamaguchi, H. (2000). A conditioning lesion promotes in vivo nerve regeneration in the contralateral sciatic nerve of rats. Biochem. Biophys. Res. Commun. 267, 715-718. doi: 10.1006/bbrc.1999.2017

Sar Shalom, H., and Yaron, A. (2014). Marking axonal growth in sensory neurons: SCG10. Exp. Neurol. 254, 68-69. doi: 10.1016/j.expneurol.2014.01.014

Schmid, A. B., Coppieters, M. W., Ruitenberg, M. J., and McLachlan, E. M. (2013). Local and remote immune-mediated inflammation after mild peripheral nerve compression in rats. J. Neuropathol. Exp. Neurol. 72, 662-680. doi: 10.1097/nen. 0b013e318298de5b

Schreyer, D. J., and Skene, J. H. P. (1993). Injury-associated induction of GAP-43 expression displays axon branch specificity in rat dorsal-root ganglion neurons. J. Neurobiol. 24, 959-970. doi: 10.1002/neu.480240709

Schwaiger, F. W., Hager, G., Schmitt, A. B., Horvat, A., Streif, R., Spitzer, C., et al. (2000). Peripheral but not central axotomy induces changes in Janus kinases (JAK) and signal transducers and activators of transcription (STAT). Eur. J. Neurosci. 12, 1165-1176. doi: 10.1046/j.1460-9568.2000.00005.x

Sheu, J. Y., Kulhanek, D. J., and Eckenstein, F. P. (2000). Differential patterns of ERK and STAT3 phosphorylation after sciatic nerve transection in the rat. Exp. Neurol. 166, 392-402. doi: 10.1006/exnr.2000.7508

Shin, J. E., and Cho, Y. (2017). Epigenetic regulation of axon regeneration after neural injury. Mol. Cells 40, 10-16. doi: 10.14348/molcells.2017.2311

Shin, J. E., Geisler, S., and DiAntonio, A. (2014). Dynamic regulation of SCG10 in regenerating axons after injury. Exp. Neurol. 252, 1-11. doi: 10.1016/j. expneurol.2013.11.007

Shin, J. E., Miller, B. R., Babetto, E., Cho, Y., Sasaki, Y., Qayum, S., et al. (2012). SCG10 is a JNK target in the axonal degeneration pathway. Proc. Natl. Acad. Sci. U S A 109, E3696-E3705. doi: 10.1073/pnas.1216204109

Skene, J. H. P., and Willard, M. (1981). Axonally transported proteins associated with axon growth in rabbit central and peripheral nervous systems. J. Cell Biol. 89, 96-103. doi: 10.1083/jcb.89.1.96

Stewart, H. J. S., Cowen, T., Curtis, R., Wilkin, G. P., Mirsky, R., and Jessen, K. R. (1992). GAP-43 immunoreactivity is widespread in the autonomic neurons and sensory neurons of the rat. Neuroscience 47, 673-684. doi: 10.1016/03064522(92)90175-2

Sugiura, Y., and Mori, N. (1995). SCG10 expresses growth-associated manner in developing rat brain, but shows a different pattern to p19 stathmin or GAP-43. Dev. Brain Res. 90, 73-91. doi: 10.1016/0165-3806(96)83488-2
Tararuk, T., Ostman, N., Li, W. R., Björkblom, B., Padzik, A., Zdrojewska, J., et al. (2006). JNK1 phosphorylation of SCG10 determines microtubule dynamics and axodendritic length. J. Cell Biol. 173, 265-277. doi: 10.1083/jcb.200511055

Temporin, K., Tanaka, H., Kuroda, Y., Okada, K., Yachi, K., Moritomo, H., et al. (2008). IL-1 $\beta$ promotes neurite outgrowth by deactivating RhoA via p38 MAPK pathway. Biochem. Biophys. Res. Commun. 365, 375-380. doi: 10.1016/j.bbrc. 2007.10.198

Tsai, S. Y., Yang, L. Y., Wu, C. H., Chang, S. F., Hsu, C. Y., Wei, C. P., et al. (2007). Injury-induced janus kinase/protein kinase $\mathrm{C}$-dependent phosphorylation of growth-associated protein 43 and signal transducer and activator of transcription 3 for neurite growth in dorsal root ganglion. J. Neurosci. Res. 85 , 321-331. doi: 10.1002/jnr.21119

Valakh, V., Frey, E., Babetto, E., Walker, L. J., and DiAntonio, A. (2015). Cytoskeletal disruption activates the DLK/JNK pathway, which promotes axonal regeneration and mimics a preconditioning injury. Neurobiol. Dis. 77, 13-25. doi: 10.1016/j.nbd.2015.02.014

Verma, P., Chierzi, S., Codd, A. M., Campbell, D. S., Meyer, R. L., Holt, C. E., et al. (2005). Axonal protein synthesis and degradation are necessary for efficient growth cone regeneration. J. Neurosci. 25, 331-342. doi: 10.1523/JNEUROSCI. 3073-04.2005

Wells, M. R., Vaidya, U., and Schwartz, J. P. (1994). Bilateral phasic increases in dorsal-root ganglia nerve growth-factor synthesis after unilateral sciatic-nerve crush. Exp. Brain Res. 101, 53-58. doi: 10.1007/bf00243216

Yamaguchi, H., Ochi, M., Mori, R., Ryoke, K., Yamamoto, S., Iwata, A., et al. (1999). Unilateral sciatic nerve injury stimulates contralateral nerve regeneration. Neuroreport 10, 1359-1362. doi: 10.1097/00001756-19990426000037

Zamboni, L., and Demartin, C. (1967). Buffered picric acid-formaldehyde-a new rapid fixative for electron microscopy. J. Cell Biol. 35:A148.

Zigmond, R. E. (2011). gp130 cytokines are positive signals triggering changes in gene expression and axon outgrowth in peripheral neurons following injury. Front. Mol. Neurosci. 4:62. doi: 10.3389/fnmol.2011.00062

Zigmond, R. E. (2012). Cytokines that promote nerve regeneration. Exp. Neurol. 238, 101-106. doi: 10.1016/j.expneurol.2012.08.017

Conflict of Interest Statement: The authors declare that the research was conducted in the absence of any commercial or financial relationships that could be construed as a potential conflict of interest.

Copyright (C) 2019 Dubový, Klusáková, Hradilová-Svízenská, Brázda, Kohoutková and Joukal. This is an open-access article distributed under the terms of the Creative Commons Attribution License (CC BY). The use, distribution or reproduction in other forums is permitted, provided the original author(s) and the copyright owner(s) are credited and that the original publication in this journal is cited, in accordance with accepted academic practice. No use, distribution or reproduction is permitted which does not comply with these terms. 